\title{
Identification and analysis of MKK and MPK gene families in canola (Brassica napus L.)
}

\author{
Wanwan Liang ${ }^{1 \dagger}$, Bo Yang ${ }^{1 \dagger}$, Bao-Jun Yu' , Zili Zhou' ${ }^{1}$, Cui Li ${ }^{1}$, Ming Jia', Yun Sun ${ }^{1}$, Yue Zhang ${ }^{1}$, Feifei Wu',
} Hanfeng Zhang ${ }^{1}$, Boya Wang ${ }^{1}$, Michael K Deyholos ${ }^{2}$ and Yuan-Qing Jiang ${ }^{1 *}$

\begin{abstract}
Background: Eukaryotic mitogen-activated protein kinase (MAPK/MPK) signaling cascades transduce and amplify environmental signals via three types of reversibly phosphorylated kinases to activate defense gene expression. Canola (oilseed rape, Brassica napus) is a major crop in temperate regions. Identification and characterization of MAPK and MAPK kinases (MAPKK/MKK) of canola will help to elucidate their role in responses to abiotic and biotic stresses.

Results: We describe the identification and analysis of seven MKK (BnaMKK) and 12 MPK (BnaMPK) members from canola. Sequence alignments and phylogenetic analyses of the predicted amino acid sequences of BnaMKKs and BnaMPKs classified them into four different groups. We also examined the subcellular localization of four and two members of BnaMKK and BnaMPK gene families, respectively, using green fluorescent protein (GFP) and, found GFP signals in both nuclei and cytoplasm. Furthermore, we identified several interesting interaction pairs through yeast two-hybrid (Y2H) analysis of interactions between BnaMKKs and BnaMPKs, as well as BnaMPK and BnaWRKYs. We defined contiguous signaling modules including BnaMKK9-BnaMPK1/2-BnaWRKY53, BnaMKK2/4/5-BnaMPK3/ 6-BnaWRKY20/26 and BnaMKK9-BnaMPK5/9/19/20. Of these, several interactions had not been previously described in any species. Selected interactions were validated in vivo by a bimolecular fluorescence complementation (BiFC) assay. Transcriptional responses of a subset of canola MKK and MPK genes to stimuli including fungal pathogens, hormones and abiotic stress treatments were analyzed through real-time RT-PCR and we identified a few of BnaMKKs and BnaMPKs responding to salicylic acid (SA), oxalic acid (OA), Sclerotinia sclerotiorum or other stress conditions. Comparisons of expression patterns of putative orthologs in canola and Arabidopsis showed that transcript expression patterns were generally conserved, with some differences suggestive of sub-functionalization.

Conclusions: We identified seven MKK and 12 MPK genes from canola and examined their phylogenetic relationships, transcript expression patterns, subcellular localization, and protein-protein interactions. Not all expression patterns and interactions were conserved between canola and Arabidopsis, highlighting the limitations of drawing inferences about crops from model species. The data presented here provide the first systematic description of MKK-MPK-WRKY signaling modules in canola and will further improve our understanding of defense responses in general and provide a basis for future crop improvement.
\end{abstract}

Keywords: Abiotic stress, Biotic stress, Brassica napus, MKK, MPK, Sclerotinia sclerotiorum, WRKY

\footnotetext{
* Correspondence: jiangyq@nwafu.edu.cn

†Equal contributors

${ }^{1}$ State Key Laboratory of Crop Stress Biology for Arid Areas and College of Life Sciences, Northwest A \& F University, Yangling, Shaanxi 712100, China Full list of author information is available at the end of the article
}

\section{() Biomed Central}

(c) 2013 Liang et al.; licensee BioMed Central Ltd. This is an Open Access article distributed under the terms of the Creative Commons Attribution License (http://creativecommons.org/licenses/by/2.0), which permits unrestricted use, distribution, and reproduction in any medium, provided the original work is properly cited. 


\section{Background}

Environmental stresses including salinity, extreme temperatures, limited water availability, and fungal pathogens deeply limit agricultural productivity. Alleviating these problems and ensuring sustainable agricultural development is a priority for plant biologists. During their evolution, plants have acquired a complex defense system to responsd to adverse environmental conditions. The mitogen-activated protein kinase (MAPK/MPK) cascades, which are composed of three groups of protein kinases: MAPKK kinases (MAPKKKs, MAP3Ks, or MEKKs), MAPK kinase (MAPKKs, MAP2Ks, MKK or MEKs) and MAPK/MPKs, are conserved in eukaryotes through evolution and operate as sequential signal transducers via phosphorylation to amplify, channel and integrate information from the cellular environment to the transcriptional response and metabolic response centers $[1,2]$.

In Arabidopsis, there are 80 MAPKKK, 10 MKK and 20 MPK genes [3,4]. The ten MKK genes in Arabidopsis may activate different MPKs and hence may integrate cross-talk of different signaling pathways. MKKs can be classified into four major groups (A, B, C and D) based on their S/TxxxxxS/T consensus domain and "D sites" [4]. AtMKK1, -2, -4 and -5 have been reported to play roles in plant stress responses [5-8]. The MPK families also have four major groups (A, B, C and D) based on their conserved T-D/E -Y motif with groups A, B, C harboring T-E-Y while group D has T-D-Y motif [4]. Arabidopsis MPK3, -4 and -6 , which are in group A and $B$ respectively, are involved in many diverse processes including developmental processes and stress responses [9-12].

WRKY transcription factors (TFs) are important transcriptional regulators that modulate immune responses and abiotic stresses [13]. Emerging evidence demonstrates that group I WRKY transcription factors, which contain a conserved SP cluster and/or D domain motif in their Nterminal regions, could be activated by MAPK-dependent phosphorylation, suggesting post-translational regulation of WRKY transcription factors [14]. So far, several MAPKKK-MKK-MPK and MPK-WRKY modules and their functions have been well-studied. For instance, Arabidopsis MPK4 is phosphorylated by upstream MKK1/ 2 and MEKK1 and, once phosphorylated, it phosphorylates and activates MAP kinase 4 substrate 1 (MKS1), which then triggers the dissociation of the inactivated form of MPK4-MKS1-WRKY33 to release MKS1WRKY33 [12,15-18] The released MKS1-WRKY33 can then enter the nucleus to initiate the transcription of downstream PHYTOALEXIN DEFICIENT3 (PAD3), required for the antimicrobial camalexin production $[12,18]$. On the other hand, AtMPK3 and -6 are activated by upstream MKK4 and -5 to phosphorylate WRKY33 and, through transcriptional regulation of the camalexin biosynthetic genes PAD2 and PAD3, confer resistance to fungal pathogens [19]. A more recent report provided direct evidence that AtWRKY33 is phosphorylated by AtMPK3 and AtMPK6 in vivo in responses to Botrytis cinerea infection [20], suggesting that phosphorylation of WRKY transcription factors by MPKs could be an important means to transduce the signal to nuclei.

Expression of a constitutively active AtMPK4 (CAMPK4) reduced SA accumulation and defense resistance against bacteria Pseudomonas syringae pv tomato strain DC3000 (Pst DC3000) [21]. Moreover, pathogenassociated molecular pattern (PAMP)-triggered reactive oxygen species (ROS) were also repressed in CA-MPK4 plants, which is different from the effects regulated by either MPK4 or the loss-of-function $m p k 4$ mutant [21]. Arabidopsis MEK1-MPK6 was also involved in abscisic acid (ABA) and sugar signaling in the process of seed germination, as the induction of NCED3 and $A B A 2$ by sugar was abolished in the double mutant mek1/mpk6 [22]. AtMPK3/6-AtWRKY33 regulates 1-amino-cyclopropane-1-carboxylic acid synthase (ACS) activity, a rate-limiting step in ethylene biosynthesis pathway, at both transcriptional and protein stability levels in plant immune responses to B. cinerea [23]. AtMPK3 and -6 are also involved in stomatal dynamics and development [24]. Stomatal closure caused by drought and other stresses is mediated by the phytohormone ABA and $\mathrm{H}_{2} \mathrm{O}_{2}$ and, this process also requires the participation of the AtMKK1 and AtMPK3 and -6 [25]. AtMKK3 could act upstream of group C MPKs (MPK1, -2, -7 and -14) to regulate the downstream pathogenesis-related (PR) genes since ProPR1::GUS expression was enhanced by the co-expression of AtMKK3/AtMPK7. AtMKK3overexpression lines with increased PR gene expression are tolerant while the knock-out $m k k 3$ lines are susceptible to Pst DC3000 [26].

MAPK signaling cascades have also been reported to play important roles in other plant species. A MAPK from rice (Oryza sativa), BWMK1, can phosphorylate a transcription factor, OsEREBP1, and overexpressing $B W M K 1$ in tobacco enhanced expression of many pathogenesisrelated genes with enhanced resistance to pathogens [27]. OsMAPK5 is induced by various biotic (pathogen infection) and abiotic (wounding, drought, salt, and cold) stresses; however, overexpression or RNAi-mediated suppression demonstrated that OsMAPK5 can positively regulate drought, salt, and cold tolerance while negatively modulating PR gene expression and resistance to fungal (Magnaporthe grisea) and bacterial (Burkholderia glumae) pathogens [28]. A more recent report shows that OsMKK6 phosphorylates OsMPK3 and these constitute a moderately $\left(12^{\circ} \mathrm{C}\right)$ but not a severely $\left(4^{\circ} \mathrm{C}\right)$ low temperature signalling pathway [29]. In Nicotiana 
benthamiana and Nicotiana tabacum, SA-induced protein kinase (SIPK) and wound-induced protein kinase (WIPK) are well-known to be involved into osmotic stress, wounding, and biotic stress [30,31]. In Zea mays, ZmMPK3 and -5 are reported to participate in the plant response to cold, drought, ultraviolet light and oxidative stress [32,33]. In Triticum aestivum, TaMPK3 and -6 are differentially regulated at multiple levels during compatible disease interactions with Mycosphaerella graminicola [34]. In Solanum lycopersicum (Lycopersicon peruvianum), LeMPK1 and LeMPK2 were activated in response to systemin, four different oligosaccharide elicitors (OEs), and UV-B radiation [35]. In Medicago sativa, SIMK, MMK2, MMK3, and SAMK were activated by excess copper or cadmium ions. Furthermore, SIMK is also activated by SIMK kinase (SIMKK). However, there are still many unknown MAPKKK-MKK-MPK modules waiting to be identified and characterized [36]. So far, no systematic investigations of MAPKK/MKK and MAPK/ MPK gene families have been reported in Brassica napus.

Canola (oilseed rape, Brassica napus L.) is a major oil crop in temperate regions and its quality and quantity are often limited by adverse environmental stresses including fungal pathogens. The fungal disease sclerotinia stem rot, caused by S. sclerotiorum (Lib.) de Bary, is a particular problem in canola [37]. Due to the lack of resistant germplasm, and the fact that the resistance is possibly mediated by multiple genes, it is necessary to identify and explore the genes that contribute to plant resistance against $S$. sclerotiorum [38-42]. In our previous transcriptional study of canola infected with S. sclerotiorum, we identified several WRKY genes and MPK3, $-4,-6$ and -17 as well as several MKK and MAPKKK genes that are responsive to $S$. sclerotiorum infection [43]. Further, from canola, we identified 46 and cloned 38 WRKY genes and studied them [44]. However the biological significance and functions of most of the canola MKK and MPK genes, except MPK4, have not been described so far in B. napus [11]. In the present study, we describe the identification and cloning of seven BnaMKK and 12 BnaMPK genes and, analyze them using bioinformatics and molecular and biochemical assays. We found that some of the BnaMKK and BnaMPK genes are responsive to multiple stress treatments including S. sclerotiorum, oxalic acids (OA), drought, etc. Through yeast two-hybrid $(\mathrm{Y} 2 \mathrm{H})$ assay, both BnaMKKBnaMPK and BnaMPK-BnaWRKY interactions were screened and several interesting MAPK modules were identified. Finally, comparison of expression patterns of putative orthologs from canola and Arabidopsis showed that they may be involved in different signalling pathways. Through this work, we can better understand the biotic (defense) and abiotic stress responses as well as the molecular mechanisms regualted by MKK-MPK-WRKY modules in canola. The data presented here would also lay a solid foundation for improving the tolerance of canola against S. sclerotiorum and other abiotic stresses through modulating the expression level of these genes.

\section{Results and discussion}

Identification, cloning of BnaMKK and BnaMPK genes in canola

As the first step to understand the roles of MKK and MPK signaling cascades in canola response to abiotic and biotic stresses, we aimed to clone BnaMKK and BnaMPK genes from canola. Since the sequencing of Brassica napus genome is not finished and Arabidopsis is a close relative to $B$. napus, we used the sequences of 10 MKK genes and 20 MPK genes of Arabidopsis as queries, and ran BLAST searches of the expressed sequence tag (EST) database (release 110101) of B. napus in NCBI. As a result, we identified 373 ESTs representing MPKs and 58 ESTs for MKKs (Table 1, Additional file 1: Table S1), which showed significant similarities with an e-value lower than $10^{-4}$. These ESTs were manually curated and then assembled to obtain contigs and singlets, which were then reciprocally BLAST searched against the Arabidopsis transcript database, TAIR10 (http://www.arabidopsis.org/Blast/index.jsp) to identify the putative orthologs. The names of canola MKK and MPK genes were assigned based on names of their presumptive Arabidopsis orthologs. Afterwards, the amino acids of each contig or singlet were predicted using DNAMAN or DNASTAR program. As a result, we successfully identified ESTs representing 8 BnaMKK and 18 BnaMPK genes (Table 1, Additional file 1: Table S1). To facilatate the characterization of these two gene families, we named each gene with a three-letter code starting with Bna (for B. napus) followed the family designation (MKK or MPK), and finally a number consistent with the Arabidopsis MAPK and MAPKK nomenclature (Table 1).

We used RT-PCR and RACE (rapid amplification of cDNA ends) to clone the cDNA sequences of the $B n a M K K$ and BnaMPK genes using high-fidelity polymerase. As a result, seven BnaMKK and 12 BnaMPK genes were cloned and the sequences were deposited in the GenBank (Table 1). Orthologs from different species may have similar biological functions [45]. Because functions of some MKK and MPK genes in the model plants Arabidopsis and rice have been well studied, we compared our cloned BnaMKK and BnaMPK sequences to those of Arabidopsis and rice and identified presumed orthologs in each species (Table 1).

\section{Phylogenetic analysis, multiple alignment and domain analysis of BnaMKKs and BnaMPKs}

To better understand the evolutionary history of both MKK and MPK gene families, we also retrieved MKK and MPK gene sequences from a variety of species representing major land plant lineages including the 
Table 1 BnaMPKs and BnaMKKs identified in this study

\begin{tabular}{|c|c|c|c|c|c|c|c|c|c|c|}
\hline \multirow[t]{2}{*}{ Gene } & \multirow{2}{*}{$\begin{array}{l}\text { GenBank } \\
\text { Acc No. }\end{array}$} & \multirow{2}{*}{$\begin{array}{l}\text { EST } \\
\text { count }\end{array}$} & \multirow{2}{*}{$\begin{array}{l}\text { AA } \\
\text { No. }\end{array}$} & \multirow{2}{*}{$\begin{array}{c}\mathrm{pl} \\
\text { value }\end{array}$} & \multirow{2}{*}{$\begin{array}{c}\text { Arabidopsis } \\
\text { ortholog }^{\mathrm{b}} \text { /AGI No. }\end{array}$} & \multirow[t]{2}{*}{ Rice ortholog $\mathrm{b} /$ locus } & \multicolumn{3}{|c|}{ Subcellular localization $^{c}$} & \multirow[b]{2}{*}{ experimenta } \\
\hline & & & & & & & PSORT & CELLO & $\overline{\text { ESLPred }}$ & \\
\hline BnaMKK1 & JQ708028 & 3 & 356 & 6.75 & AtMKK1/ At4g26070 & OsMKK1/Os06g05520.1 & nuc & nuc & cyt & \\
\hline BnaMKK2 & JQ708029 & 3 & 365 & 5.99 & AtMKK2/ At4g29810 & N/A & cyt & cyt, nuc & cyt & cyt, nuc \\
\hline BnaMKK3 & JQ708030 & 3 & 519 & 5.58 & AtMKK3/ At5g40440 & OsMKK3/Os06g27890.1 & cyt, nuc & cyt, pm & cyt & cyt, nuc \\
\hline BnaMKK4 & JQ708031 & 4 & 357 & 9.2 & AtMKK4 /At1g51660 & OsMKK5/Os06g09180.1 & nuc, cyt & nuc & nuc & cyt, nuc \\
\hline BnaMKK5 & KC246595 & 2 & 333 & 8.89 & AtMKK5/ At3g21220 & OsMKK4/Os02g54600.1 & mit & nuc & nuc & \\
\hline BnaMKK6 & JQ708032 & 2 & 357 & 6.19 & AtMKK6/ At5g56580 & OsMKK6/Os01g32660.1 & cyt & nuc, cyt & cyt & \\
\hline BnaMKK8 & No & 1 & & & AtMKK7/ At5g56581 & & & & & \\
\hline BnaMKK9 & JQ708033 & 1 & 307 & 8.22 & AtMKK9/ At1g73500 & N/A & chl, nuc & mit & cyt & \\
\hline BnaMPK1 & JQ708034 & 2 & 371 & 6 & AtMPK1/ At1g10210 & OsMPK7/Os06g48590.1 & nuc & cyt & cyt & \\
\hline BnaMPK2 & JQ708038 & 5 & 374 & 6.6 & AtMPK2/ At1g59580 & N/A & & & cyt & \\
\hline BnaMPK3 & JQ708040 & 4 & 371 & 6 & AtMPK3/ At3g45640 & OsMPK3/Os03g17700.1 & nuc, cyt & cyt & nuc & cyt, nuc \\
\hline BnaMPK4 & JQ708041 & 7 & 374 & 6.06 & AtMPK4/ At4g01370 & OsMPK4/Os10g38950.1 & cyt & cyt & nuc & \\
\hline BnaMPK5 & JQ708042 & 3 & 374 & 5.7 & AtMPK5/ At4g11330 & OsMPK2/Os08g06060.1 & cyt & cyt & nuc & cyt, nuc \\
\hline BnaMPK6 & JQ708043 & 6 & 396 & 5.19 & AtMPK6/ At2g43790 & OsMPK6/Os06g06090.1 & nuc & cyt & nuc & cyt, nuc \\
\hline BnaMPK7 & No & 13 & & & AtMPK7/ At2g18170 & & & & & \\
\hline BnaMPK8 & JQ708044 & 12 & 582 & 6.65 & AtMPK8/ At1g18150 & N/A & nuc & cyt & nuc & \\
\hline BnaMPK9 & JQ708045 & 1 & 502 & 8.42 & AtMPK9/ At3g18040 & OsMPK17-1/Os06g49430.1 & cyt, nuc & cyt & cyt & cyt, nuc \\
\hline BnaMPK10 & No & 2 & & & AtMPK10/At3g59790 & & & & & \\
\hline BnaMPK12 & No & 4 & & & AtMPK12/At2g46070 & & & & & \\
\hline BnaMPK13 & No & 1 & & & AtMPK13/At1g07880 & & & & & \\
\hline BnaMPK15 & No & 1 & & & AtMPK15/At1g73670 & & & & & \\
\hline BnaMPK16 & JQ708035 & 12 & 559 & 8.8 & AtMPK16/At5g19010 & OsMPK/Os05g05160.1 & cyt & cyt & cyt & \\
\hline BnaMPK17 & JQ708036 & 10 & 488 & 6.83 & AtMPK17/At2g01450 & N/A & cyt & cyt & cyt & \\
\hline BnaMPK18 & No & 32 & & & AtMPK18/At1g53510 & & & & & \\
\hline BnaMPK19 & JQ708037 & 7 & 600 & 9.2 & AtMPK19/At3g14720 & N/A & cyt & pp,cyt & cyt & \\
\hline BnaMPK20 & JQ708039 & 10 & 612 & 9.23 & AtMPK20/At2g42880 & OsMPK20-1/Os01g43910.1 & cyt & cyt & mit & \\
\hline
\end{tabular}

${ }^{a}$ The full-length cDNA was cloned and deposited in GenBank. No, cloning not sucessful.

${ }^{b}$ Putative orthologs were identified by InParanoid (http://inparanoid.sbc.su.se/cgi-bin/index.cgi) with a maximum score. N/A, homolog but not ortholog was identified.

'Subcellular localizations are predicted using three different programs, one is PSORT (http://psort.nibb.ac.jp), the second is CELLO v2.5 (http://cello.life.nctu.edu.tw) and the third is ESLPred (http://www.imtech.res.in/raghava/eslpred/index.html). nuc-nuclear, cyt-cytoplasm,pp-perplasm, mit-mitochondria,chl-chloroplast,and pm-plasma membrane.

bryophyte Physcomitrella patens (Pp), the lycophyte Selaginella moellendorffii (Sm), and several mono- and eudicotyledonous angiosperms, namely: the eudicots Arabidopsis thaliana (At), Vitis vinifera (Vv), Populus trichocarpa (Pt) and Glycine $\max (\mathrm{Gm})$; and the monocots Oryza sativa (Os), Sorghum bicolor (Sb), Brachypodium distachyon (Bd), and Zea mays (Zm). By searching the green alga Chlamydomonas reinhartdii (Cr) genome in Phytozome v9.0, we also identified two MKK genes, whose protein sequences showed great similarity to AtMKK3 and AtMKK6, respectively (Additional file 2: Table S2). Furthermore, we also identified an MKK gene from a marine green alga Ostreococcus tauri $(\mathrm{Ot})$, which is the world's smallest free-living eukaryote known to date [46]. It should be noted that based on evidence from ESTs, the size of the canola MKK protein family is roughly comparable to that of either Arabidopsis, rice or poplar, although not all MKK genes have been cloned from canola. On the other hand, the green alga $C$. reinhartdii and lower land plants $P$. patens and S. moellendorffii apparently evolved a smaller set of MKKs relative to the flowering plants, which indicates an expansion of MKK gene family after the divergence of flowering plants from the other lineages.

The amino acid sequences of MKKs collected from 21 species, together with our BnaMKK sequences, were used to construct a phylogenetic tree using the maximum parsimony (MP) method (Figure 1, Additional file 3: Figure S1). The OtMKK was used to root the tree. As shown by the tree's topology, the MKK proteins from 


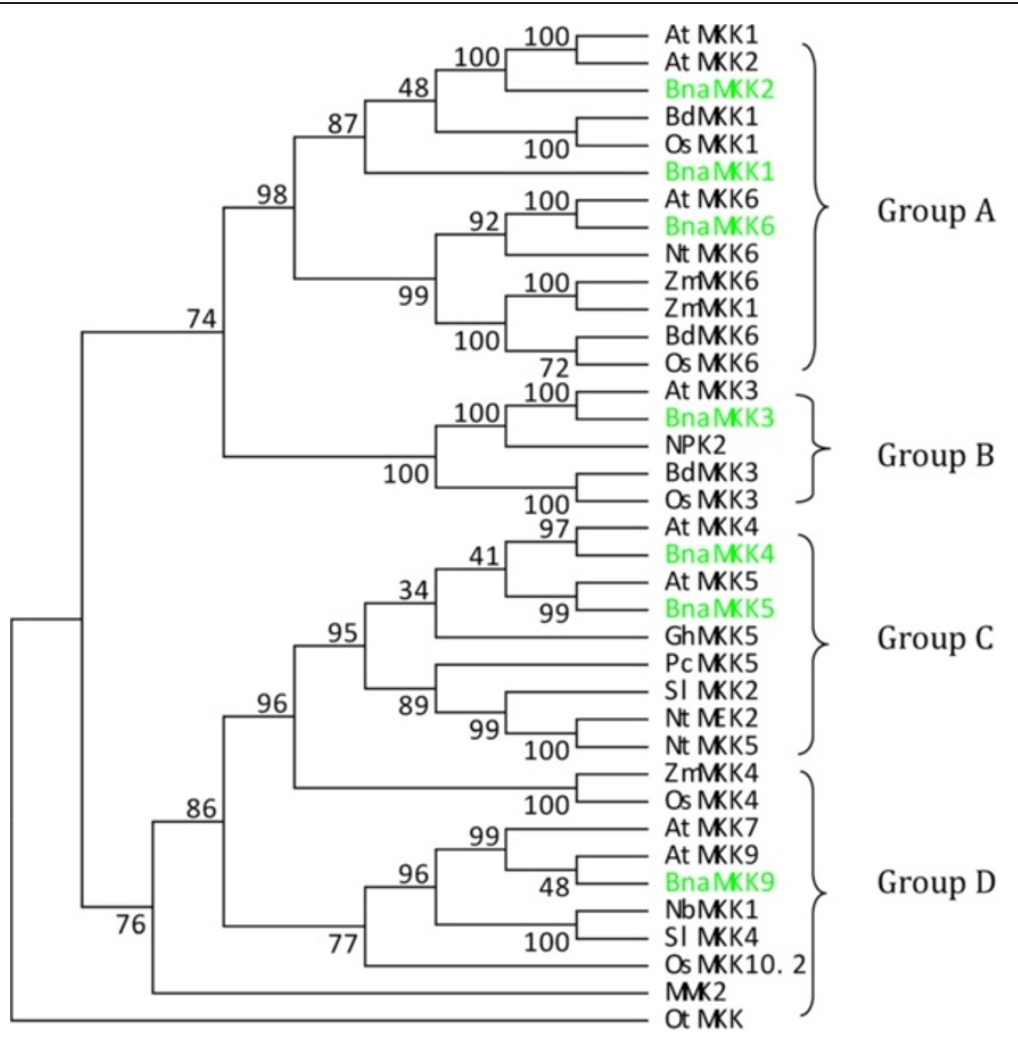

Figure 1 Phylogenetic analysis of MKKs and domain analysis of seven BnaMKKs. Phylogenetic relationship of BnaMKKs with MKKs from other species. The respective MKK proteins are depicted by a two to three-letter code denoting the species in combination with numbers indicating the specific MKK gene from each species. The phylogenetic tree was reconstructed using the maximum parsimony method of MEGA5.1 program. The green highlighted proteins are from canola. The analysis involved 39 amino acid sequences. There are a total of 594 positions in the final dataset. OtMKK, an MKK from the marine green alga Ostreococcus tauri (Ot) was used to root the tree. The numbers on the nodes are percentages from a bootstrap analysis of 500 replicates. At, A. thaliana; Bd, Brachypodium distachyon; Bna, Brassica napus; $\mathrm{Cr}$, Chlamydomonas reinhartdii; Gh, G. hirsutum; Nt, N. tabacum; Ot, O. tauri; Os, O. sativa; PC,P. crispum; SI, S. lycopersicum and Zm, Z. mays.

various species could be divided into four major groups, each supported by highly significant bootstrap values. The seven canola MKKs were distributed in each of the four groups, with BnaMKK1, -2 and -6 belonging to Group A, BnaMKK3 Group B, BnaMKK4 and -5 Group C, and BnaMKK9 Group D (Figure 1), which is consistent with previous phylogenetic analysis of MKKs from Arabidopsis, rice and poplar [1]. Furthermore, the seven BnaMKK members were always clustered in the same subgroup closely with AtMKK orthologs, which was expected for genes from these two representatives of the Brassicaceae family. The multiple alignment of the collected BnaMKKs showed that they each have a putative MPK docking sites (D sites) at the $\mathrm{N}$ terminus, characterized by a cluster of basic residues ( $\mathrm{R}$ or $\mathrm{K}$ ) $\mathrm{N}$-terminal to hydrophobic residues (L or I) (Figure 2A), as reported for the MKKs from model species [4]. These BnaMKK proteins also contain a highly conserved phosphorylation site, which has a consensus sequence $\mathrm{S} / \mathrm{TxxxxxS/T}$, as previously identified in Arabidopsis MKK proteins (Figure 2A).
The conserved motifs among the BnaMKKs were further analyzed using the MEME4 program, and a schematic of the motifs is presented (Figure 2B, Additional file 4: Figure $\mathrm{S} 2 \mathrm{a}$ and b). Seven motifs were conserved in all BnaMKKs identified, while motifs 7, 8, 9 and 10 were group-specific. Group A (BnaMKK1, -2 and -6) has motif 9 in the $\mathrm{N}$-terminal sequence especially, while only group C (BnaMKK4 and -5) and D (BnaMKK9) had motif 10. BnaMKK2 had an extra motif 6 in the $C$ terminal region. In addition, we found that BnaMKK3 had a long C-terminal region and a similar observation was also made with BdMKK3 [47]. The motifs from MEME analysis were further annotated by comparisons with known protein kinase motifs/domains (http:// prosite.expasy.org/scanprosite/). We noted that motif 1 contained both IiHrDLKpsNLLV, which is the active-site signature of serine/threonine protein kinases, and $\mathrm{S} /$ TXXXXS/T, which is the consensus sequence [4]. Motif 5 contains protein kinases ATP-binding signature, IGKGSSGVVQIVqhkwtgqf, in which a glycine-rich loop 

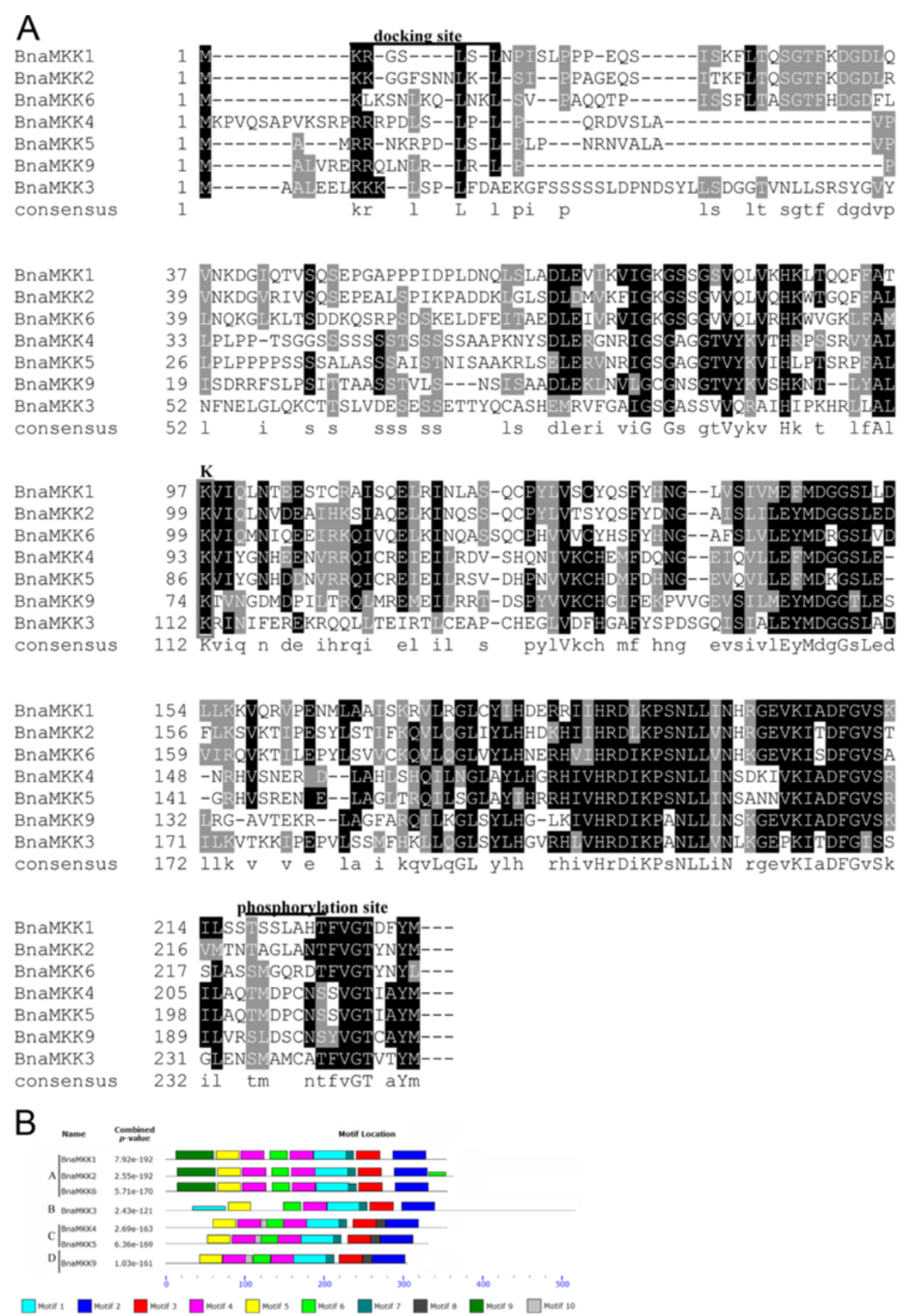

Figure 2 Sequence analysis of BnaMKKs. (A) Partial amino acid alignment of the seven BnaMKK proteins. Multiple sequence alignment was performed using the ClustalX1.83 and illustrated by BOXSHADE (http://www.ch.embnet.org/software/BOX_form.html). Identical amino acids are shaded in black, and similar amino acids are shaded in gray. The docking and phosphorylation sites are indicated by overbars, with the lysine (K) necessary for the kinase activity is boxed. (B) Schematic diagram of amino acid motifs of BnaMKKs. Motif analysis was determined by using MEME4.0 program as described in Methods. Different colors of the boxes represent 10 motifs in the corresponding position of each BnaMKK proteins. The information of 10 motifs was illustrated in Additional file 4: Figure S2.

(G-x-G-x-x-G) is required for ATP binding. Motif 7 (VGTxxYMSPER) is the conserved signature in the catalytic domains as described previously [4].

To further explore the evolutionary history of the MAPK family in plants, we retrieved MPK sequences from a various plant species (Additional file 2: Table S2). We identified a single MPK gene from $O$. tauri $(O t)$ and used its protein sequence to root a bootstrapped consensus tree. We performed a phylogenetic analysis of the 12 BnaMPKs in the context of MPK proteins 
retrieved from a representative selection of species (Figure 3) and then in all the MPK sequences collected (Additional file 5: Figure S3). We found that the identified 12 BnaMPKs could be assigned unambiguously to four separate clades, together with 20 AtMPKs [4]. Group A included BnaMPK3 and -6, group B includes BnaMPK4 and -5 and, group $C$ includes BnaMPK1 and -2 , whereas six BnaMPKs belong to D, which are BnaMPK8, $-9,-16$,

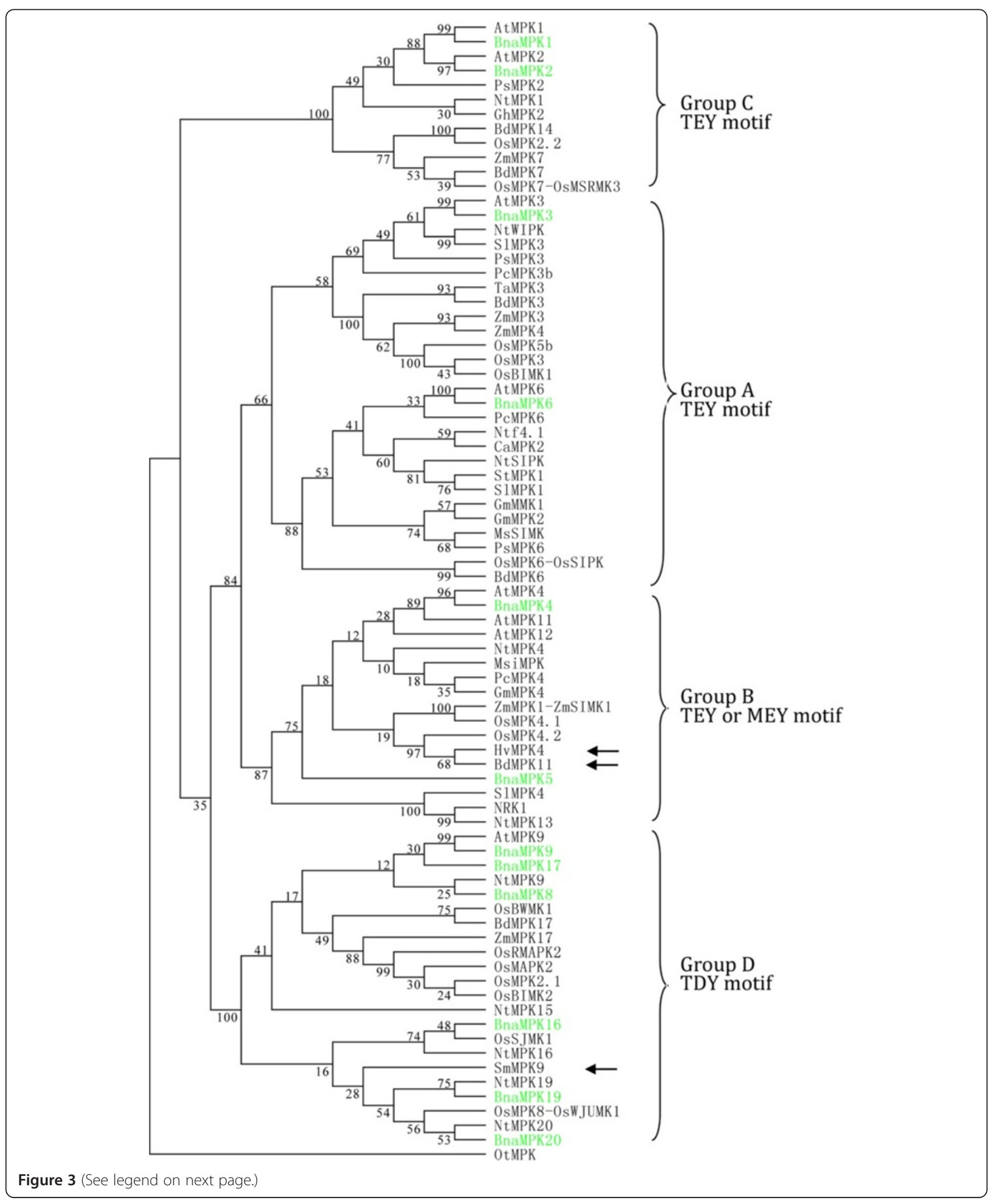


(See figure on previous page.)

Figure 3 Phylogenetic analysis of MPKs and domain analysis of 12 BnaMPKs. Phylogenetic relationship of BnaMPKs with MKKs from other species. The respective MPK proteins are depicted by a two to three-letter code denoting the species in combination with numbers representing the exact MKK from this species. The phylogenetic tree was reconstructed through maximum parsimony method of MEGA5.1 program. The green highlighted proteins are BnaMPKs. The analysis involved 85 amino acid sequences. There are a total of 752 positions in the final dataset. The numbers on the nodes are percentages from a bootstrap analysis of 500 replicates. The atypical MEY motif in the activation loop of a few MPKs is marked by arrows. OtMPK is used as the outgroup. At, A. thaliana; Bd, Brachypodium distachyon; Bna, Brassica napus; Ca, C. annuum; Gm, G. max; Gh, G. hirsutum; Ms, M. sativa; Msi, Malus sieversii; Nt,N. tabacum; Os,O. sativa; Ot,O. tauri; Pc,P. crispum; Ps, P. sativum; Pt, P. trichocarpa; SI, S. lycopersicum; Ta,T. aestivum and Zm, Z. mays.

$-17,-19$ and -20 . Most MPKs are classified into group D instead of group A, B or C, which is partially in agreement with previous study in rice [48]. This indicates that gene loss may have occurred in groups A to $\mathrm{C}$, after the monocot-dicot divergence [48]. As expected, most of the BnaMPKs were clustered more closely with Arabidopsis than those of other species.

MPKs are located at the terminus of prototypical sequential cascades, and can be phosphorylated and therefore activated by MKKs via dual phosphorylation of conserved threonine and tyrosine residues in the motif TxY, which is located in the activation loop (T-loop) between subdomains VII and VIII of MPKs [4]. Each of the 12 BnaMPKs we identified possessed a TEY or TDY signature (Figure 4A). A MEY motif was identified in BdMPK11 [47] and also MPK from other species including SmMPK9, HvMPK4 and NtMPK4. Since $S$. moellendorffii is a member of an ancient vascular plant lineage that first appeared in the fossil record some 400 million years ago [49], the presence of an atypical MEYtype activation loop in this species suggests its early divergence. As can be seen from Figure 4A and Additional file 5: Figure S3, the TEY-type MPKs can be classified into three groups, A, B and C, whereas all the TDY-type MPKs form a more distinct group D, as described previously [4]. Interestingly, in the ancient unicellular green alga $O$. tauri, we also identified a single MPK, which contains a TEY motif, suggesting that the common ancestor of eukaryotic MPKs may have been of TEY type. During the long history of evolution and selective forces, MPK proteins may have acquired other types of TxY motif besides the canonical TEY motif [50]. In addition, the aforementioned three atypical MEY-type MPK proteins we identified fell into the TEY-B clade in the tree, whereas SmMPK9 was clustered together with TDY clade (Figure 4A, Additional file 5: Figure S3). Since all analyzed land plant lineages in the phylogenetic tree contained both TEY- and TDY-type MPKs, this indicated that an early gene duplication event likely generated the split between the two major canonical classes of TEYand TDY-type MPKs [51].

MPKs may contain a CD domain, defined as $[\mathrm{LH}]$ [LHY]Dxx[DE] $x x[D E] E p x C$, where $x$ means any amino acid and the two adjacent acidic residues (D and $E$ ) are critical for interacting with a cluster of basic amino acids ( $\mathrm{K}$ and $\mathrm{R}$ ) in MKKs [52]. We found that the groups A, B and $C$ of BnaMPKs possessed a $C D$ or modified $C D$ domain, while neither domain appeared in the group $D$ (Figure 4B), which is also consistent with previous findings from other species [52]. From the alignment of amino acid sequences of OsMPKs, AtMPKs and BnaMPKs, we identified the characteristic motif of the MPK family, which is defined as Fx7Rx2Rex8Hx28 Mx3Lx20QxLx6HxAx3HRDLKPxNx6C and is conserved among rice, Arabidopsis and canola as well as O. tauri, except for AtMPK1 and its ortholog BnaMPK1 (Additional file 6: Figure S4). BnaMPK1 and AtMPK1 had a Tyr (Y) instead of Phe (F) at the very beginning of the conserved motif. However, the OsMPK20.2 downloaded from the rice genome annotation project lacked this region due to an unknown reason.

The conserved motifs in the 12 BnaMPK proteins were also analyzed using MEME4, and a schematic of the motifs is presented (Figure 4B, Additional file 7: Figure S5). Seven motifs were conserved in all of the members of BnaMPK, while motifs 7, 9 and 10 are found only in some groups. For instance, Group A, B and $\mathrm{C}$ did not have motif 7 at the $\mathrm{C}$-terminal region while group $C$ and D did not have motif 10 at the $\mathrm{N}$ terminal end. Similarly, we annotated the motifs from MEME analysis and identified that eight of the ten motifs $(1,2,3,4,5,6,8$ and 10) are actually subdomains (IXI) of kinase domain of MPKs [51]. Motif 1 contains VI and VII subdomains, motif 2 is subdomain IX, motif 3 contains subdomain I harboring a glycine-rich loop (G$\mathrm{x}-\mathrm{G}-\mathrm{x}-\mathrm{x}-\mathrm{G}$ ) required for ATP binding. Motif 3 also contains subdomains II and III, which represent the activesite signature IlHrDLKpgNLLI of serine/threonine protein kinases. Motif 4 contains subdomains IV and V, motif 5 has subdomain XI and, motif 6 has subdmain VIII, which has the conserved consensus -T(D/E)Ymotif in the catalytic domain of plant kinases. Motif 8 has subdomain X. Motifs 7 and 9 contain $C D$ domain, which is (LH)DXXDE(P)X acting as a docking site for MAPKKs [4]. Consistent with our finding in Figure 4A, the motif analysis through MEME4.0 showed that Group 
$D$ did not have motif 9, which is CD domain, while Groups A, B and C had it (Figure 4B, Additional file 7: Figure S5).

From our phylogenetic analysis, multiple alignment and domain analysis of BnaMKK and BnaMPK in canola, we concluded that MKK and some of the MPK family members may be conserved among monocots while others were lost after the divergence of the monocots and dicots. In all, The MPK signaling cascades were relatively conserved during long history of evolution, and genes belonging to the same clade or group might fulfill similar functions. The phylogenetic analysis 
together with the domain motif analysis presented will facilitate the functional annotation and study of uncharacterized MKKs and MPKs.

\section{Subcellular localization of BnaMKK and BnaMPK proteins in vivo}

Previous reports of subcellular localization of MKKs and MPKs suggest specific functions of these proteins in response to diverse stimuli $[53,54]$. Subcellular localization analysis of individual MAPK modules in vivo is therefore helpful in understanding how a single MAPK component can simultaneously mediate several responses and how a single stimulus could direct several different MAPK modules to separate tasks [54]. We first used three different programs, WoLF PSORT, CELLO v2.5 and ESLPred, to predict the subcellular localization of the 7 BnaMKKs and 12 BnaMPKs (Table 1). At the same time, we used TMHMM (http://www.cbs.dtu.dk/services/TMHMM-2.0/) to predict transmembrane helices (TMHs) of these proteins, but failed to identify any TMH (data not shown). Secondly, to examine the subcellular localization of BnaMKKs and BnaMPKs in planta, we randomly selected three BnaMKK genes and four BnaMKK genes for green fluorescent protein (GFP) fusion. To this end, the coding region of BnaMKK2,-3, and -4 as well as BnaMPK3, $-5,-6,-9$ were fused to the $\mathrm{N}$-terminus of green fluorescent protein (GFP) reporter gene with a Gly-Ala rich peptide linker between CDSs and GFP in a binary vector. Then, $A$. tumefaciens cells transformed with each of these constructs and p19 protein of tomato bushy stunt virus were infiltrated into leaves of Nicotiana benthamiana. Two days later, under confocal microscope, we observed that both BnaMPKGFP and BnaMKK-GFP fusion proteins emitted green fluorescence signals in nuclei and in cytoplasm of epidermal cells of leaves (Figure 5A, C, D, F, H, I and K), suggesting that the MPK and MKK proteins were localized to the cytoplasm and are small enough to diffuse or be trafficked to the nucleus. When the leaf discs were further treated with a hyperosmotic solution $(500 \mathrm{mM}$ mannitol) for $1 \mathrm{~h}$ and the plasmolyzed tissues were examined with the same settings in confocal microscope, the GFP signals were still evident in both the cytoplasm and nuclei (Figure 5B, E, G, J and L). Taken together, these results indicated that both BnaMKKs and BnaMPKs studied are localized both in the cytoplasm and nuclei. Comparing the predicted localization patterns (Table 1) to our observations using GFP (Figure 5), the results from in silico prediction and the in vivo experimental determination were generally similar, with some exception that highlight the need to examine localization in planta.

Recent reports have provided direct evidence that specific Arabidopsis and tobacco MPKs can phosphorylate some WRKY TFs to initiate transcription of target genes $[20,55]$. TFs are usually localized in the nuclei, as demonstrated by many studies including ours [44]. Therefore, we also checked the subcellular localization of a WRKY TF, BnaWRKY20, in $N$. benthamiana and the result showed that it is localized in nuclei only (Figure $5 \mathrm{M}$ ), which is consistent with its role in gene transcription. As a control, in the $N$. benthamiana leaf cells transformed with the empty vector bearing GFP gene only, the GFP signals were also observed in both cytosol and nuclei (Figure 5EV). Therefore, the co-localization of BnaMPKs and BnaWRKYs might facilitate their interaction, phosphorylation and activation.

\section{Identification and validation of BnaMKK-BnaMPK and BnaMPK-BnaWRKY interactions}

So far, the best characterized MKK genes are from Arabidopsis, which has 10 MKK genes, and which activate different MPKs [4]. It is necessary to understand interactions between MKKs and MPKs to elucidate crosstalk between different signaling pathways. Therefore, to characterize components of the BnaMPK cascade as well as their upstream and downstream interaction partners, we used the yeast two-hybrid ( $\mathrm{Y} 2 \mathrm{H})$ system to analyze interactions between seven BnaMKKs and 12 BnaMPKs, as well as interactions between BnaMPKs and BnaWRKY TFs. To do this, seven BnaMKKs and nine BnaWRKYs (BnaWRKY1, -6, -20,-26, -28, -31, -45, -53 and -69) were individually cloned in-frame into the pGADT7 vector while the 12 BnaMPK genes were cloned into pGBKT7 vector. This strategy was chosen to avoid possible transactivation of expression of marker genes (e.g. histidine synthease gene HIS3 and $\beta$-galactosidase gene LacZ) by the TFs to be tested in Y2H. Only yeast colonies expressing both of the interacting MKKs and MPKs (or MPKs and WRKYs) could grow on the selection media SD-LTHA (synthetic dropout lacking leucine, tryptophan, histidine and adenine hemisulfate). Only MKKMPK and MPK-WRKY pairs that showed interactions were reported in Figures 6 and 7. The other combinations that did not show any interaction in our $\mathrm{Y} 2 \mathrm{H}$ assay were not presented here. The authenticity and strength of the interactions were further examined by titration assays and $\mathrm{X}$-gal staining.

Our $\mathrm{Y} 2 \mathrm{H}$ assay revealed that BnaMKK9 significantly interacted with BnaMPK1, $-2,-5,-9,-19$ and -20 , and BnaMKK9 did not show any interaction with the other seven BnaMPKs tested. On the other hand, BnaMKK4 and -5 showed interactions with BnaMPK3 and -6 (Figure 6). When these interactions were compared to those between Arabidopsis orthologs, AtMKK9 was reported to interact with AtMPK10, 17 and -20 in $\mathrm{Y} 2 \mathrm{H}$ [56]. Interaction between AtMKK9 and AtMPK5 was previously identified by protein microarray [57], 


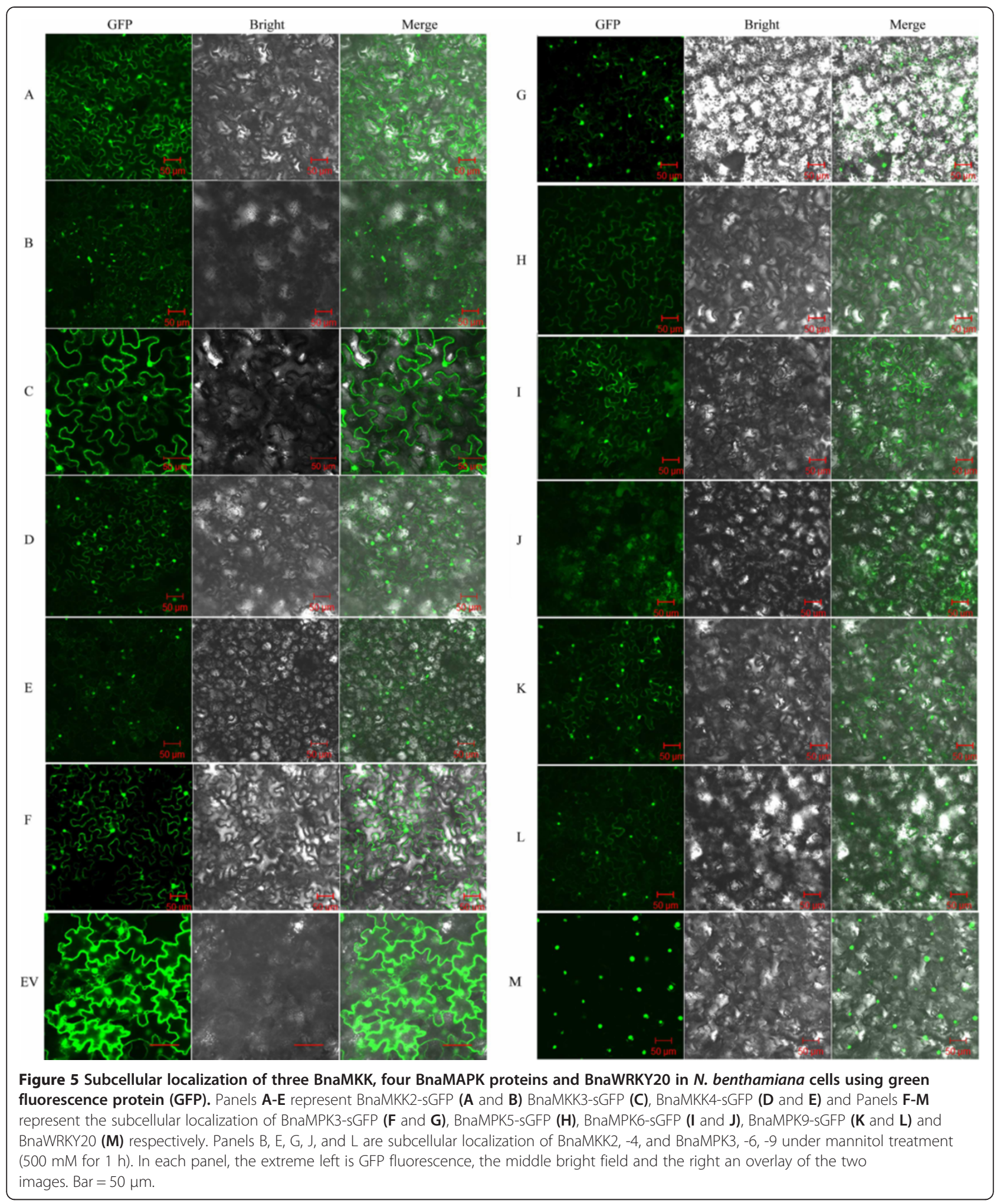

although not validated in planta yet. These differences between our observations with canola proteins and previous observations using Arabidopsis genes may be attributed to either technical or biological factors.
Different vectors and yeast strains were used in the canola and Arabidopsis experiments. The proteins may have also evolved to have different interactions in these two species. 


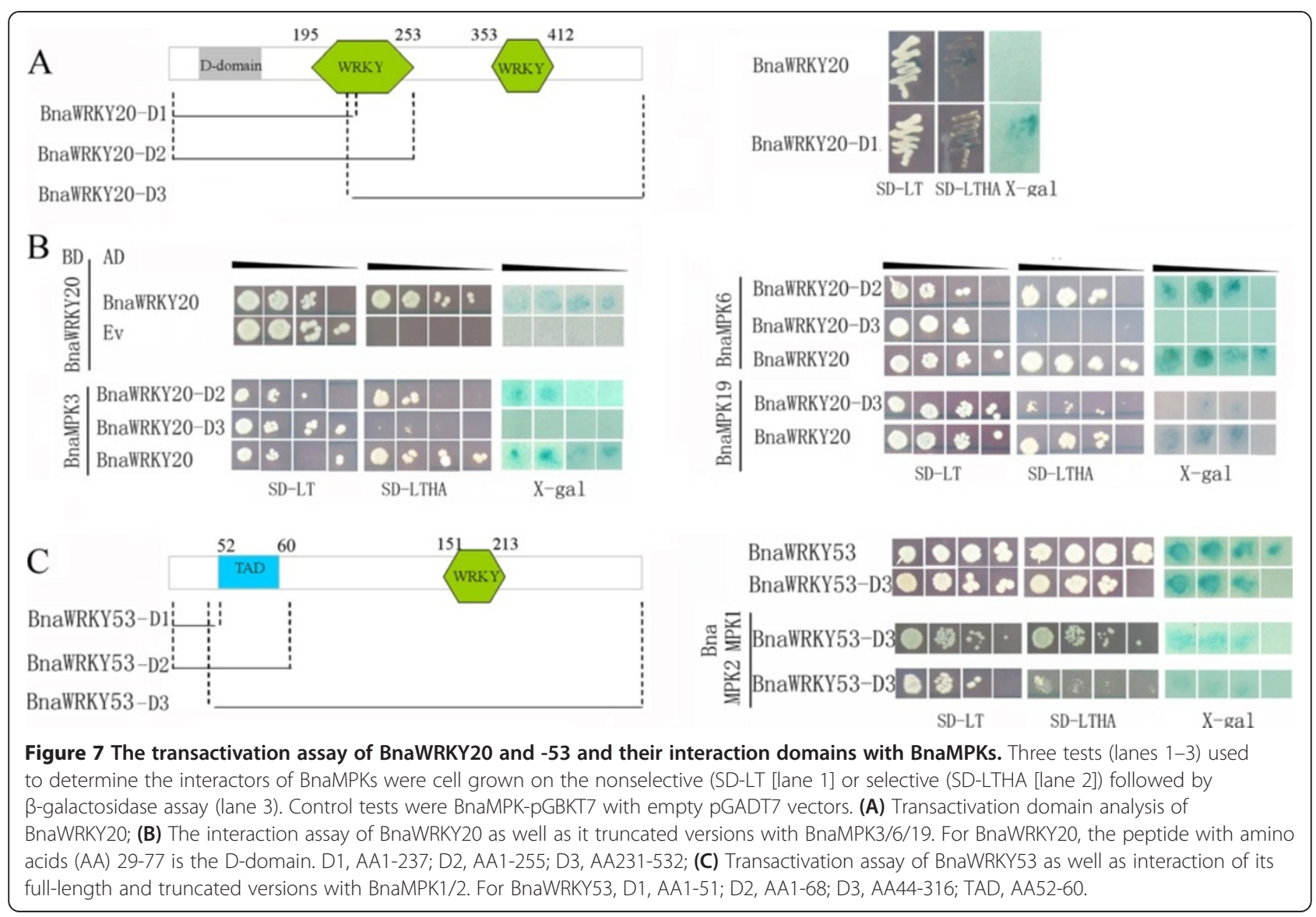


As for the downstream WRKY transcription factors that BnaMPK may phosphorylate and activate, our $\mathrm{Y} 2 \mathrm{H}$ assay showed (as also evidenced from the titration assay and Xgal assay) that BnaMPK1 and -2 significantly interacted with BnaWRKY53 and BnaMKK9 (Figure 6A). This is in agreement with a previous study showing interaction between AtMPK2 and AtWRKY53 through protein microarray profiling [57], although the interaction needs to be validated in planta. A previous study demonstrated that AtMEKK1 could directly regulate AtWRKY53 in the process of leaf senescence [58]. Based on the protein microarray analysis, validated in $N$. benthamiana, AtWRKY53 was phosphorylated when co-expressed with the AtMKK7/ AtMPK7 module [57]. Whether this is true also for the presumed canola orthologs is yet to be confirmed, since no cDNA or EST has been identified for BnaMKK7. Nevertheless, our study indicates that both BnaMPK1 and -2 could act upstream of BnaWRKY53 and may therefore phosphorylate and activate BnaWRKY53 to transcribe downstream target genes in canola.

To confirm the interaction of BnaMKK9 and BnaMPK1, -2 as well as the interaction between BnaMPK1, -2 and BnaWRKY53, we performed in vivo bimolecular fluorescence complementation (BiFC) analysis. Yellow fluorescence signals appeared in infiltrated cells when BnaMKK9 with BnaMPK1 were co-expressed and YFP signals also appeared in nuclei when BnaMPK1 and BnaWRKY53 were co-infiltrated (Figure 8A, B). As a control, BnaMPK1 with empty vector did not show any YFP signal (Figure $8 \mathrm{C}$ ). As we identified that BnaMKK9 interacted with BnaMPK1, -2, $-5,-9,19$ and -20 (Figure 6A, E), and only BnaMPK1/2 and -19 were found to interact with BnaWRKY53 and BnaWRKY20, respectively (Figure 6A, C), the downstream targets of BnaMPK5, -9 , or -20 are still waiting to be identified through $\mathrm{Y} 2 \mathrm{H}$ screening of cDNA libraries. As BnaMPK19 was identified to interact with both upstream BanMKK9 and downstream BnaWRKY20 (Figure 6C, E), these three components BnaMKK9-MPK19-WRKY20 may constitute a signaling module to regulate responses to endogeneous and external stimuli.

As MAPKs are recognized as nodal points in many signaling pathways, further efforts should include identification of their direct substrates and systematic observations of MAPK signaling regulation in specific environmental or developmental contexts. In Arabidopsis, only a few of the 10 MKKs and 20 MPKs as well as their targets have been characterized in vivo. For instance, overexpression of AtMKK7 and AtMKK9 accelerate cell death in $N$. benthamiana [57] and AtMPK5 may affect synergism of the JA and SA signaling networks [59]. Since AtWRKY53 regulates leaf senescence and defense against $P$. syringae $[60,61]$ and AtMKK9 was reported to be involved in the biosynthesis of ethylene and camlexin, and also participates in the process of leaf senescence [62,63], we hypothesized that the BnaMKK9-BnaMPK1/2-BnaWRKY53 module we identified in canola may function in leaf senescence, hormone signaling and defense responses. However, although AtMKK9 regulates ethylene signaling through interactions with MPK3 and -6 [64], we did not indentify any interaction of BnaMKK9 with BnaMPK3 or -6 . This may reflect an inherent difference in signaling pathways between Arabidopsis and canola, and highlights the necessity to study the MAPK-mediated signaling pathways in canola separately, instead of simply inferring these networks from results in Arabidopsis.

On the other hand, BnaMPK3 and -6 interacted significantly with both upstream BnaMKK2, -4 and -5 and with downstream BnaWRKY20, -26 as shown from the titration and $\mathrm{X}$-gal assays (Figure 6B, C and D). To confirm the authenticity of the interactions between MKK4/5 and $\mathrm{MPK} 3 / 6$, we alternated the $\mathrm{Y} 2 \mathrm{H}$ vectors for $M K K 4 / 5$ and $M P K 3 / 6$, which is to say that BnaMKK4/5 were cloned into pGBKT7 and BnaMPK3/6 were cloned into pGADT7 vector. The interactions between BnaMKK4/5 and BnaMPK3/ 6 were maintained even after the vectors were switched (data not shown). Similarly, in Arabidopsis, MKK4 interacts with MPK3/6 and MKK5 with MPK6 in Y2H [56]. Moreover, in protein microarrays, Arabidopsis MKK4 interacts with MPK6, and MKK5 with MPK3/6 [57], although these interactions need to be confirmed using a complementary technique, and their biological significance elucidated. Further comparisons revealed that AtMKK2 was reported to interact strongly with MPK4, $-6,-10$ and -11 but weakly with MPK13, and no interaction between AtMKK2 and MPK3 was detected [56]. Hence, $\mathrm{Y} 2 \mathrm{H}$ analysis of interactions between BnaMKK4/5 and BnaMPK3/6 are consistent with previous results with Arabidopsis orthologs. However, the interactions between MKK2 and MPK3/6 were different, despite the relatively close evolutionary relationship between Arabidopsis and canola.

As for the possible signaling components downstream of MPK3 and -6 in Arabidopsis, the function of the module of MEKK1-MKK4/5-MPK3/6 and their downstream targets WRKY22/29 are well known in regulating plant immune responses against $P$. syringae [7]. Another study showed that AtMPK3/MPK6 activation could impede the infection of the fungal pathogen Botrytis cinerea while the resistance was compromised in mpk3 and mpk6 mutants and, AtMPK3 and 6 function upstream of Phytoalexin Deficient $(P A D) 2$ and -3 to regulate the biosynthesis of camalexin and hence the plant immune response [19]. In Arabidopsis plants expressing constitutively active MKK2 (MKK2-EE), following challenge with $P$. syringae, the levels of JA, ET and SA were elevated rapidly, and the infection was therefore impeded. The downstream targets of MKK2 are MPK4 and 6, and their transcript levels were also increased and the activity of MPK4 was triggered in MKK2-EE infected with P. syringae [65]. However, whether BnaMPK3 and -6 


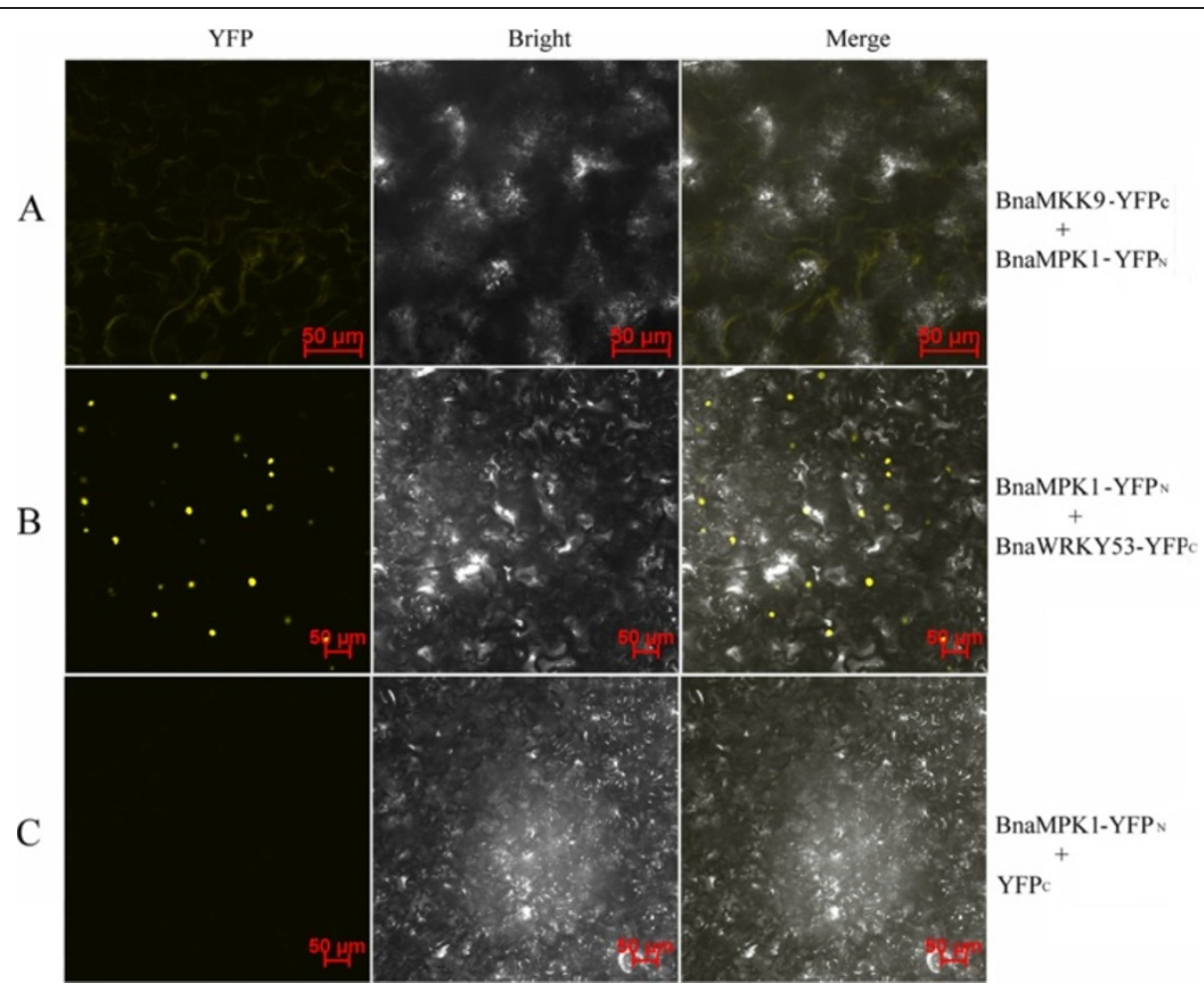

Figure 8 In vivo BiFC analysis of BnaMPK1 interacting proteins BnaMKK9 and BnaWRKY53 in co-transformed in $N$. benthamiana leaf epidermal cells. BnaMPK1 was fused to N-terminal while (A) BnaMKK9 and (B) BnaWRKY53 were fused to C-terminal halves of YFP, individually. (C) A vector control with no protein fusion to the C-terminal YFP is also shown. The fluorescence of YFP was observed by confocal laser microscopy. Bar $=50 \mu \mathrm{m}$.

and their associated complicated linear MPK pathway function in regulating plant immune response and other processes needs to be further analyzed.

As one group of the direct targets of MAP kinases in plants, WRKY TFs play very important roles in modulating abiotic and biotic stresses responses. One of our previous reports has identified 46 WRKY TF genes from canola and partially characterized some of them in the context of hormone responses and fungal pathogen challenge [44]. To test the interactions between the 12 BnaMPKs and nine BnaWRKYs, we used Y2H. The results demonstrated that BnaMPK3/6 interacted strongly with BnaWRKY20/26 (Figure 6B, C). BnaWRKY20 and BnaWRKY26 belong to Group I of the WRKY gene family. We also found that the amino acid sequences of both BnaWRKY20 and -26 have proline-directed serines (SP cluster) and a docking domain (D domain) at their $\mathrm{N}$-termini, which are widely conserved in group I WRKYs [14]. The SP clusters are putative phosphorylation targets of MAPKs and the D domain is a cluster of basic residues followed by LxL motif $\left((\mathrm{K} / \mathrm{R})_{1-2^{-} \times}{ }_{2-6^{-}}\right.$ $\mathrm{L} / \mathrm{I}-\mathrm{X}-\mathrm{L} / \mathrm{I})$ and it is thought to select the interacting MAPKs and determine the substrate specificity of MAPKs in mammals and yeast [66], however the significance in plants still await to be tested. The identification of two upstream MAP kinases, BnaMPK3 and BnaMPK6, would provide a clue that activities of BnaWRKY20 and -26 are likely regulated by them. So far, the ortholog of BnaWRKY26 in Arabidopsis together with two other Group I members AtWRKY25 and -33 has been shown to regulate salinity responses and thermotolerance $[67,68]$. However, the upstream regulators, especially MAP kinase (s), of AtWRKY26 in response to either salinity or heat have not been reported. Moreover, the functions of WRKY20 and WRKY26 in relation to signaling in other stresses have not yet been reported. Finally, for some of the nine BnaWRKYs screened, we did not detect any interacting BnaMPKs, which could be due to at least two causes. One possible explanation is that other unidentified BnaMPKs may interact with them. Alternatively, other types of protein kinases, like calcium-dependent protein kinases (CDPKs/CPKs) may act upstream of these BnaWRKYs [69].

Through $\mathrm{Y} 2 \mathrm{H}$, we also experimentally determined that BnaMKK1 interacts with BnaMPK6 (Figure 6D), while in Arabidopsis MKK1 interacts with MPK4/11 only [56], further highlighting the limitations of Arabidopsis as a model for canola. In Arabidopsis, MKK1 and MPK6 both participate in $\mathrm{ABA}$ and sugar signaling in the process of seed germination since loss of function mutants of $M K K 1$, MPK6 or both are resistant to ABA or glucose treatments while on the contrary, the overexpression of $A t M K K 1$ or 
AtMPK6 are sensitive to these treatments [22]. Our study of BnaMKK1 interactions with BnaMPK6 presented here suggests that these may play similar roles in seed germination, however these need to be characterized in detail.

In summary, from our yeast two-hybrid and BiFC assays, two complete signaling modules could be inferred, among which one is BnaMKK9-BnaMPK1/2-BnaWRKY53 and the other BnaMKK2/4/5-BnaMPK3/6-BnaWRKY20/26 (Figure 9). Other MAPK modules have also been identified, with the upstream BnaMKK9 and downstream MAP kinases, BnaMPK5, -9, -19 and -20 (Figure 9). Among these identified interaction pairs, quite a few have not been reported even in Arabidopsis suggesting that the different combination of BnaMKK and BnaMPKs, as well as BnaMPKs and BnaWRKYs very likely mediate the responses to different external and internal stimuli in canola.

\section{Transactivation and truncation assays of BnaMPK- interacting BnaWRKY transcription factors}

To further characterize the biological significance of the aforementioned BnaMPK-interacting BnaWRKY TFs, we performed transactivation assays of BnaWRKY20, -26 and -53 in yeast (Figure 7). We cloned BnaWRKY genes into the vector pGBKT7. The results showed that neither BnaWRKY20 (Figure 7A) nor BnaWRKY26 (data not shown), showed transactivation activity, suggesting that they may be transcriptional repressors. In contrast, BnaWRKY53 showed transactivation activity in yeast (Figure 7C), indicating it is a transcriptional activator. Sequence analysis of the BnaWRKY53 protein sequence showed that it contained a transactivation domain (TAD) near it $\mathrm{N}$-terminus (Figure $7 \mathrm{C}$ ), while no such domain was detected in BnaWRKY20 (Figure 7A) or -26. Although yeast colonies expressing full length BnaWRKY20 protein could not grow on the selective medium SD-LTHA, colonies expressing a truncated version of BnaWRKY20, namely BnaWRKY20-D1, in which the C-terminal amino acids between 238 and 532 were deleted, grew on SDLTHA (Figure 7A), suggesting that the sequence of amino acids between 238 and 532 of BnaWRKY20 has the transcriptional repression activity. Interestingly, we also found that BnaWRKY20 can interact with itself in yeast, indicating that it may homodimerize as part of its normal function (Figure 7B). A previous study also demonstrated that another three Arabidopsis WRKY TFs, WRKY18, -40 and -60 , which belong to Group IIa, are transcriptional repressors and interact with each other in yeast [71].

To further identify the interacting regions of BnaWRKY20 with BnaMPK3, -6 and -19 , we constructed a series of truncated versions of BnaWRKY20 and analyzed their interaction with BnaMPK3, -6 and -19 . As shown in Figure 7B, yeast colonies expressing full length of BnaWRKY20 and BnaMPK3, or -6, -19 could grow on selective media SD-LTHA, and yeast colonies expressing BnaWRKY20-D2, BnaMPK3 or -6 could also grow on selection medium SD-LTHA. These results imply that both BnaMPK3 and -6 can interact with both BnaWRKY20 and truncated BnaWRKY20 containing the N-terminal 255 amino acids. As for BnaMPK19, it can only interact with the full length and the $\mathrm{C}$-terminal peptide (amino acids of 231 to 532) of BnaWRKY20 (Figure 7C). For BnaWRKY53, we also constructed three deletion plasmids and, $\mathrm{Y} 2 \mathrm{H}$ assay demonstrated that both BnaMPK1 and BnaMPK2 interacted with BnaWRKY53-D3, which harbors amino acids of 51-317 (Figure 7C), but did not interact with BnaWRKY53-D1 or BnaWRKY53-D2 (data not shown).

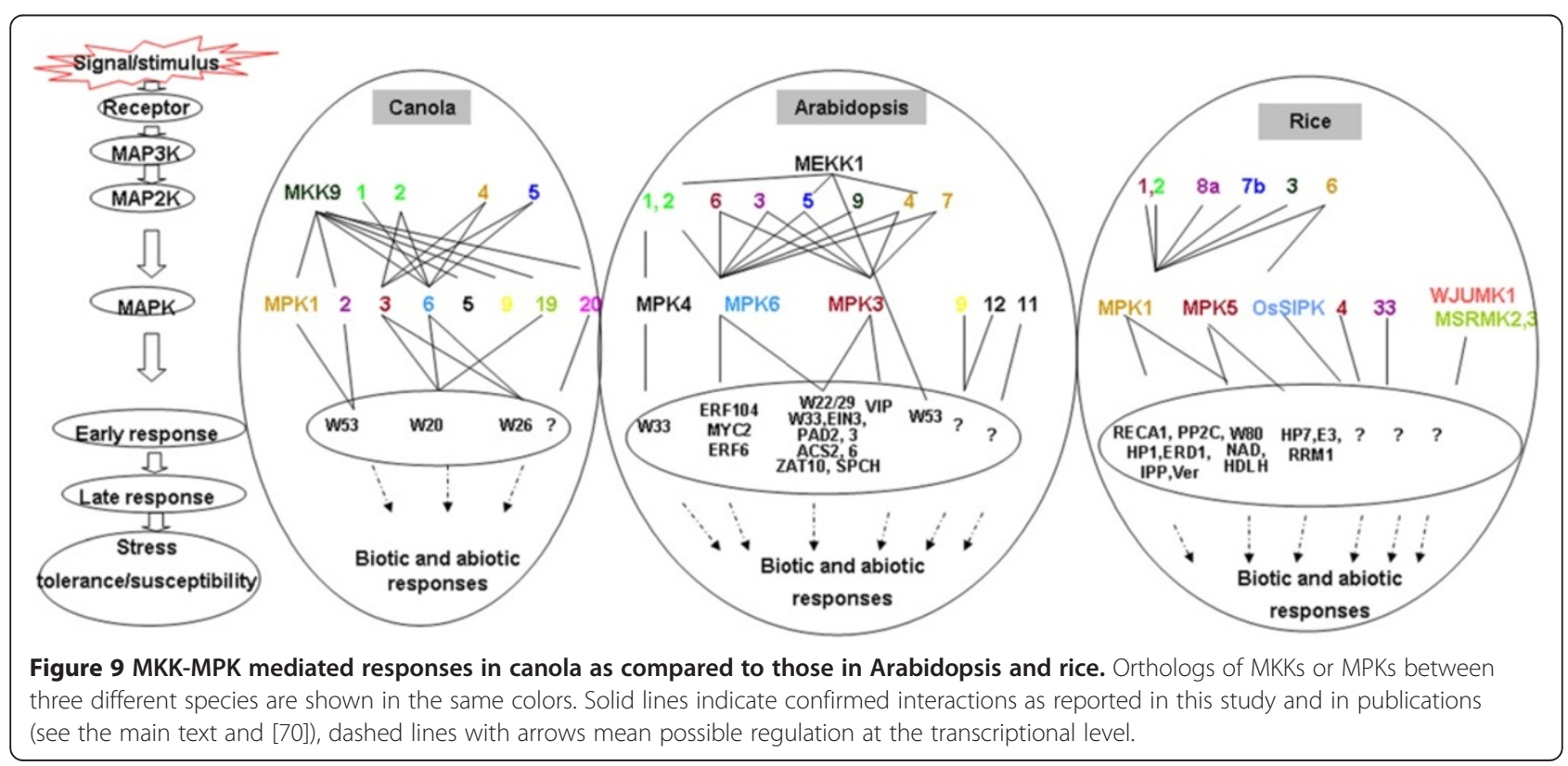




\section{Expression analyses of BnaMKK and BnaMPK genes in response to abiotic and biotic stresses}

Accumulating evidence has demonstrated that members of MKK and MPK gene families in Arabidopsis, rice as well as other species function in responses to abiotic and biotic stresses [2,72]. Therefore, we examined transcript expression profiles of canola BnaMKK and BnaMPK using quantitative real-time RT-PCR (qRT-PCR) under different abiotic, biotic stress treatments as well as hormone stimuli. We treated 18-day-old canola seedlings with jasmonic acid (JA), salicylic acid (SA), abscisic acid (ABA), methyl viologen (MV), ACC, $\mathrm{NaCl}$, cold, oxalic acid (OA) and S. sclerotiorum. Among these, JA and SA are two known plant defense hormones, with JA mediating responses to necrotrophs [73] and SA regulating responses to biotrophic pathogens and systemic acquired resistance [74]. ABA is a well-known stress hormone involved in abiotic stress such as salinity and drought. However, recent evidences showed that ABA also has a prominent role in biotic stress [75]. MV was used to induce oxidative burst or accumulation of reactive oxygen species (ROS) in plant cells, which is similar to the effect generated by hydrogen peroxide $\left(\mathrm{H}_{2} \mathrm{O}_{2}\right)$, but more stable than $\mathrm{H}_{2} \mathrm{O}_{2}$. ACC was used to mock the effect of ethylene, which is an important hormone in many processes including mediating different types of induced resistance [76]. OA is a virulence factor secreted by the necrotrophic pathogen S. sclerotiorum [77-79]. Recent studies have shown that controlling OA production might help to protect canola plants against $S$. sclerotiorum $[42,80,81]$.

Statistical analyses of qRT-PCR data from three independent biological replicates demonstrated that transcripts of four out of five (80\%) BnaMKK genes and six out of nine (67\%) BnaMPK genes were significantly induced or repressed by at least one stress condition of cold, OA, SA and S. sclerotiorum (Figures 10 and 11). As for BnaMKK genes, four genes (BnaMKK1, -2, -4, and -9, $80 \%)$ were responsive to $\mathrm{SA}$ and only three genes (BnaMKK1, -2, and -4, 40\%) were responsive to $S$. sclerotiorum. One gene (BnaMKK4, 20\%) is significantly induced by MV at $6 \mathrm{~h}$ while another gene (BnaMKK2, $20 \%)$ was responsive to OA. As for BnaMPKs genes, three genes (BnaMPK3, -5 , and $-8,33.3 \%$ ) were responsive to SA, four (BnaMPK2, $-5,-8$ and $-9,66.7 \%$ ) were responsive to $S$. sclerotiorum and, two (BnaMPK3 and $-8,22.2 \%$ ) were responsive OA treatment (Figure 11). BnaMPK1, -2, -6 and -8 were also responsive to SA treatment, although the changes are not significantly (Figure $11, p<0.05$ ). The results we presented here show that BnaMKK and BnaMPK genes are generally responsive to biotic and abiotic stress treatments and may play an important role in responses to environmental stresses and pathogens. These results also highlight the extent of cross-talk that exists between abiotic and biotic stresses.

\section{Comparison of the expression patterns of BnaMKKs and BnaMPKs with those of orthologs in Arabidopsis}

To further compare the expression profiles and roles of $B n a M K K$ and BnaMPK with their orthologs in Arabidopsis, we extracted the expression profiles of AtMKK and AtMPK genes from Genevestigator (https:// www.genevestigator.com/gv/plant.jsp). We found that on the whole, the responses of $M K K$ and $M P K$ genes between canola and Arabidopsis plants at the transcript levels to a variety of abiotic and biotic stress treatments were similar and showed a large degree of correlation (Additional file 8: Table S3). For instance, AtMKK1, -2, -4, and -9 are all induced by SA treatment, which is consistent with our observation on BnaMKK1, -2, -4, and -9 (Figure 10). AtMKK9 is induced by JA, SA, ABA, oxidative stress, salt and cold as well as a necrotrophic fungus, $B$. cinerea, which implies that AtMKK9 may be involved in response to diverse stresses, while its ortholog in canola is induced significantly only by SA and cold (Figure 10), which suggests that AtMKK9 and BnaMKK9 may play different roles in two different plant species. AtMKK9 was reported to be involved in the biosynthesis of ethylene and camlexin, and also participate the process of leaf senescence $[62,63]$. We also noted that BnaMPK19 was induced by cold, which is consistent with the expression pattern of its presumed ortholog in Arabidopsis (Figure 10, Additional file 8: Table S3). AtMKK1 and AtMPKK2 with their upstream AtMEKK1 and downstream AtMPK4 function together in regulating plant innate immunity [5]. BnaMKK3 did not respond to any treatment tested in our project, however, its ortholog AtMKK3 was reported to participate in JA signaling pathway together with AtMPK6 [82].

In summary, after comparing the transcript expression profiles of MKK and MPK genes between canola and Arabidopsis, we found that most, but not all of them show similar responses towards various abiotic and biotic stress treatments. However, some differences between the BnaMKKs and BnaMPKs and their Arabidopsis counterparts were noticed. The function of some members of the MKK and MPK gene families in stress-related hormone signaling, for instance, $M K K 6, M P K 1,-2,-5,-8,-16,-17,-19$ and -20 even in Arabidopsis still wait to be studied. Furthermore, what is the function of BnaMPK1 and -2 and are they involved in early signaling process leading to leaf senescence modulated by BnaWRKY53? Based on our recent results from a systematic functional analysis of canola MAPKKK genes, we found that BnaMKK9 is a target for at least three different BnaMAPKKKs (Sun et al, manuscript in preparation), suggesting BnaMKK9 may be a convergence point in the MAPK signaling cascade. Therefore, BnaMKK9 may be integrated in certain signaling pathway in canola defense response and this need to be elucidated through a more detailed functional analysis. 


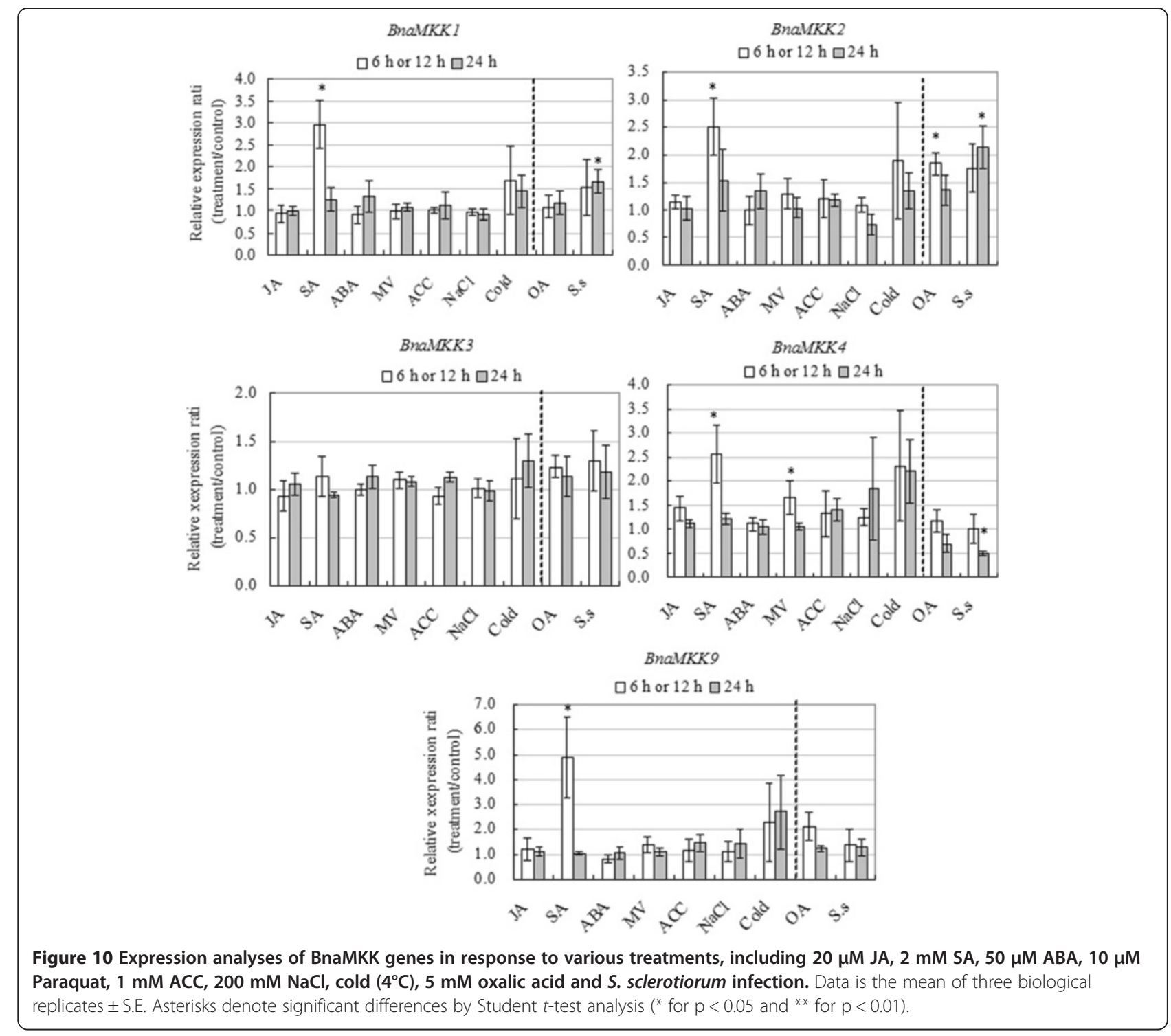

We were also curious to know whether each component of the linear modules we identified showed similar transcriptional responses to the hormone and stress treatments in a similar trend. We found that components of the same module generally showed similar responses to a given treatment, although some differences were observed. For instance, in the BnaMKK9BnaMPK5 module, both genes were induced by SA (Figures 10 and 11). In the module of BnaMKK9BnaMPK1/2-BnaWRKY53, the transcript levels of BnaMKK9, BnaMPK1, -2 and BnaWRKY53 were increased by SA while changes in BnaMPK2 and BnaWRKY53 were not significant (Figure 10, Additional file 8: Table S3). On the other hand, some components of the same module showed different responses. For instance, in the module of BnaMKK9-BnaMPK5 and BnaMKK9-BnaMPK19, BnaMKK9 transcripts did not show any response towards $S$. sclerotiorum infection at either 6 or $24 \mathrm{~h}$, while BnaMPK5 was induced and BnaMPK19 repressed by $S$. sclerotiorum inoculation (Figure 11). In the module of BnaMKK9-BnaMPK1/2BnaWRKY53, BnaMPK2 was induced while BnaWRKY53 repressed by $S$. sclerotiorum (Figure 12). Our results suggest that BnaMKK9 may regulate different MPK components in fulfilling different functions in responses to diverse stresses. In addition, in the module of BnaMKK2/ 4/5-BnaMPK3/6-BnaWRKY20/26, BnaMKK2, -4, BnaMPK3 and -6 were all induced by SA (Figures 10 and 11), which suggests that this module might be integrated in the SA signaling pathway. Surprisingly, the transcript level changes of each component in this module were different when challenged by S. sclerotiorum, BnaMKK4 was repressed (Figure 11) while BnaMPK3 and the downstream target BnaWRKY20 were both induced by 

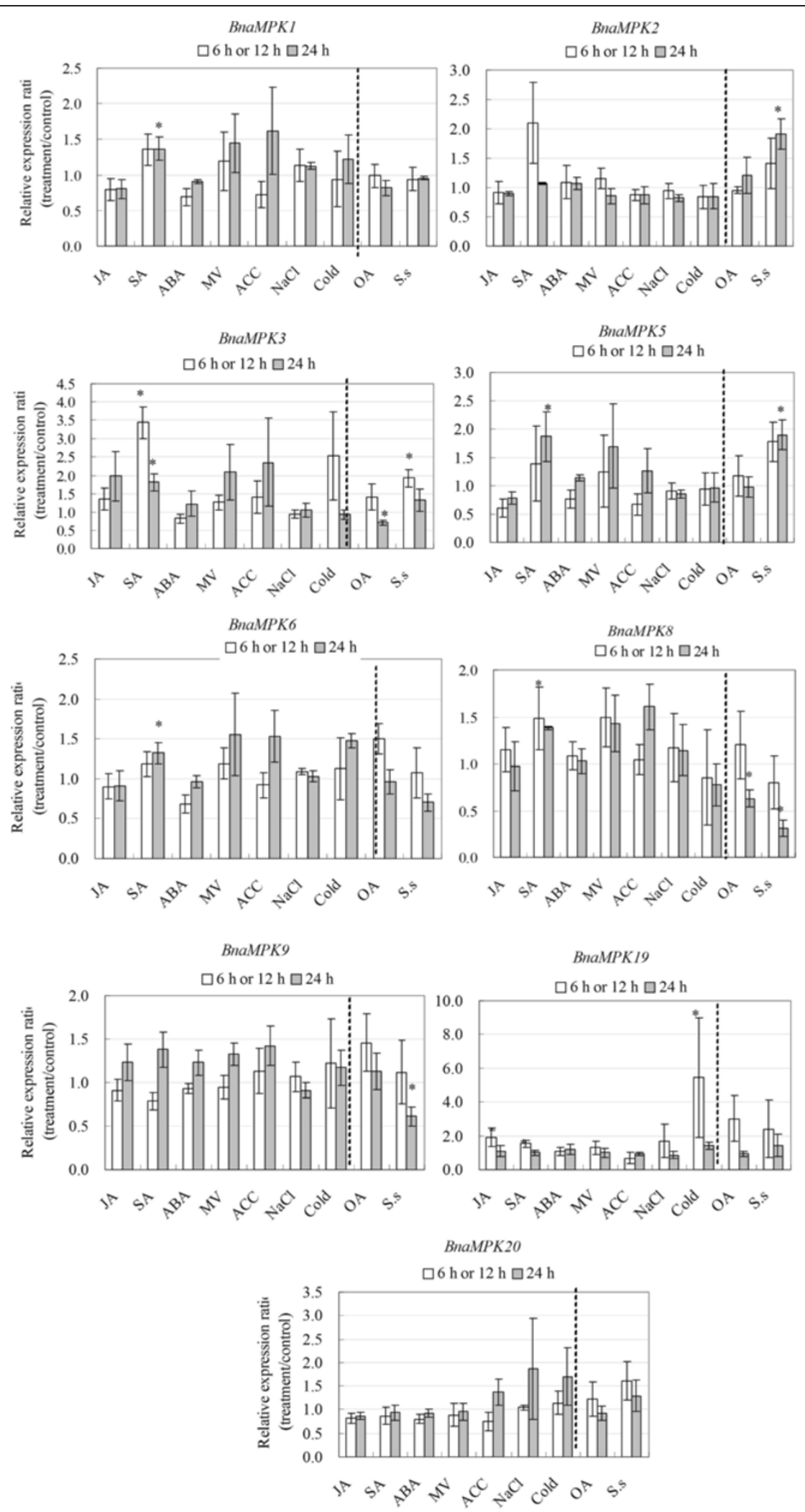

Figure 11 Expression analyses of BnaMPK genes in response to various treatments, including $20 \mu \mathrm{M} \mathrm{JA}, 2 \mathrm{mM} \mathrm{SA}, 50 \mu \mathrm{M}$ ABA, $10 \mu \mathrm{M}$ Paraquat, $1 \mathrm{mM} \mathrm{ACC}, 200 \mathrm{mM} \mathrm{NaCl}$, cold $\left(4^{\circ} \mathrm{C}\right), 5 \mathrm{mM}$ oxalic acid and S. sclerotiorum infection. Data is the mean of three biological replicates \pm S.E. Asterisks denote significant differences by Student $t$-test analysis ( ${ }^{*}$ for $p<0.05$ and ${ }^{* *}$ for $p<0.01$ ). 


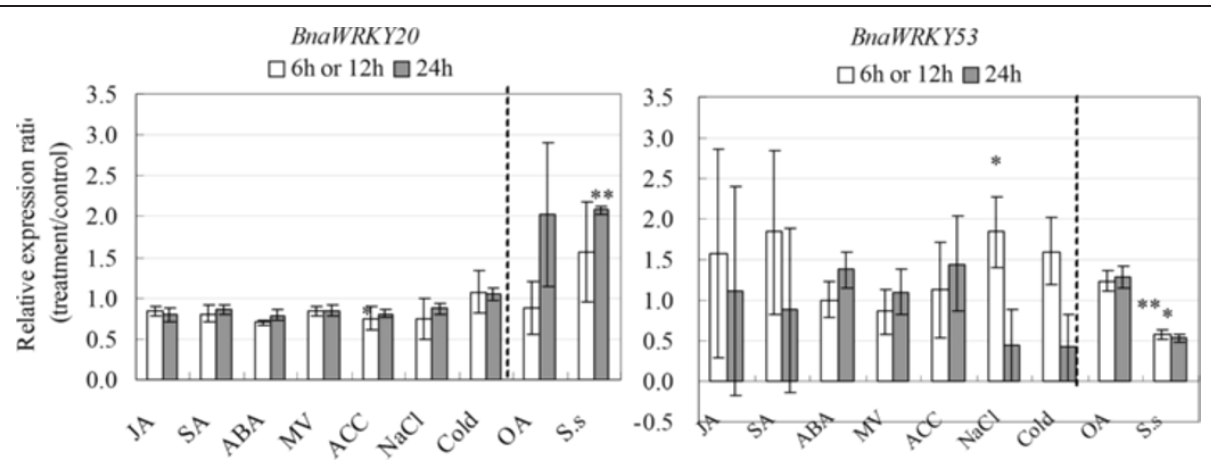

Figure 12 Transcript expression of BnaWRKY53 in response to various treatments, including $20 \mu \mathrm{M} \mathrm{JA}, 2 \mathrm{mM} \mathrm{SA}, 50 \mu \mathrm{M}$ ABA, $10 \mu \mathrm{M}$ Paraquat, $1 \mathrm{mM} \mathrm{ACC}, 200 \mathrm{mM} \mathrm{NaCl}$, cold $\left(4^{\circ} \mathrm{C}\right), 5 \mathrm{mM}$ oxalic acid and S. sclerotiorum infection. Data is the mean of three biological replicates \pm S.E. Asterisks denote significant differences by Student $t$-test analysis ( ${ }^{*}$ for $p<0.05$ and ${ }^{* *}$ for $p<0.01$ ).

S. sclerotiorum (Figures 11 and 12). As for modules of BnaMKK1/2-BnaMPK19, we observed that BnaMKK1 and -2 were induced by SA, S. sclerotiorum and OA (Figure 10) and BnaMPK19 is induced by cold only (Figure 11). Interestingly, no significant changes on the mRNA abundance of the above selected BnaMKK or BnaMPK genes were observed when JA was applied to seedlings. All of the data suggest that post-transcriptional regulation and post-translational modifications including phosphorylation might play an important role in modulating their kinase activities beyond the transcriptional regulation.

\section{Conclusions}

In the present study, we identified and cloned seven BnaMKK and 12 BnaMPK genes from an important crop, canola (oilseed rape) and reconstructed the phylogeny of both families based on major land plant lineages. Our study complements several previous analyses which inferred the phylogenetics based on limited land plant lineages $[1,4,50]$. Since the core of MAPK signaling in all eukaryotes is organized in three-tiered modules, it was necessary to dissect the subcellular localization of individual MKK and MAPK members, as well as their interactors to dismantle the complexity of plant MAPK modules and networks. Our GFP-based translational fusions confirmed the cytoplasmic and nuclear subcellular localizations of both BnaMKK and BnaMPK.

$\mathrm{Y} 2 \mathrm{H}$ assays and in vivo BiFC confirmation identified two linear pathways of MPK signaling modules, composed of BnaMKK-BnaMPK-BnaWRKY and some new interacting partners (Figure 9). The two complete modules inferred based are BnaMKK9-BnaMPK1/2-BnaWRKY53 and BnaMKK2/4/5-BnaMPK3/6-BnaWRKY20/26 (Figure 9). Some incomplete modules were also identified, such as BnaMKK9-BnaMPK5/9/19/20 (Figure 9). Among the identified interaction pairs, many have not been previously identified, even in Arabidopsis. Analysis of their expression patterns suggested that BnaMKK9, BnaMPK1, 2 and BnaMKK2, -4, -5 and BnaMPK3 and 6 may be integrated into the SA signaling pathway to trigger defense response of canola (Figures 10 and 11). Further experiments could be carried on in the protoplast system to accurately determine the linear modules involved signaling pathways. Moreover, genetic analysis of selected MKK and MPK components of canola through overexpression and RNA interference (RNAi) techniques are underway. This work presented here will be helpful for us to better understand the roles of BnaMKK and BnaMPK genes in canola response to environmental stresses, especially the plant response to biotic stresses.

\section{Methods}

\section{Identification of MKK and MPK genes in B. napus}

$B n a M K K$ and BnaMPK gene identification was performed essentially as described previously [44]. Expressed sequence tags (ESTs) representing MKK and MPK genes were retrieved by BLAST searching the NCBI dbEST database (release 110101) using 10 AtMKKs and 20 AtMPKs as the queries, respectively. Only hits with e-values lower than $10^{-4}$ were retrieved. Altogether we obtained 18 and 84 unique ESTs representing BnaMKKs and BnaMPKs, respectively. These ESTs were further cleaned, clustered, and assembled and, each of the resultant contigs and singlets were reciprocally BLAST-searched against Arabidopsis to find the best hit, which we designated as the putative ortholog. Subsequently, the amino acids were predicted using open reading frames (ORFs) in DNASTAR (DNASTAR Inc.). At this step, we identified the cDNA (mRNA) sequences of a total of eight BnaMKK and 18 BnaMPK.

\section{Plant growth and gene cloning}

Wild type canola (DH12075) plants were grown in Pindstrup soil mix (Egypt) in a glasshouse with a 
photoperiod of $16 \mathrm{~h}$ light (T5 fluorescent tubes with a light

intensity of approximately $\left.90 \mu \mathrm{E} \mathrm{m} \mathrm{m}^{-2} \mathrm{~s}^{-1}\right) / 8 \mathrm{~h}$ dark, and a temperature of $22^{\circ} \mathrm{C}$ for $7 \mathrm{~d}$. RNA was isolated from seedlings using a Plant RNA kit (Omega Bio-Tek, USA). RNA integrity was checked by electrophoresis on an agarose gel and quantified using the NanoDrop 1000 (NanoDrop Technologies, Inc., USA). First-strand cDNA was synthesized from $2.5 \mu \mathrm{g}$ of total RNA using RevertAid $\mathrm{H}$ minus reverse transcriptase (Fermentas) and oligo(dT)18 primers (Fermentas). PCR primers were designed using PrimerSelect (DNAStar Inc.) or Primer 3 (v0.4.0, http:// frodo.wi.mit.edu/) (Additional file 9: Table S4). PCR was conducted in a $50 \mu \mathrm{L}$ final volume including $0.5 \mu \mathrm{L}$ of cDNA template, $1 \times P f u$ buffer, $200 \mu \mathrm{M}$ deoxynucleotide triphosphates (dNTPs) (Promega), $400 \mathrm{nM}$ of each primer, and 1 unit of Pfu polymerase. The PCR conditions included an initial denaturation at $94^{\circ} \mathrm{C}$ for $3 \mathrm{~min}$, followed by 35 cycles of $94^{\circ} \mathrm{C}$ for $30 \mathrm{~s}, 50^{\circ} \mathrm{C}$ for $30 \mathrm{~s}, 72^{\circ} \mathrm{C}$ for 1 min per $\mathrm{kb}$, with a final extension at $72^{\circ} \mathrm{C}$ for $8 \mathrm{~min}$. RACE (rapid amplification of cDNA end) was performed as described [44]. PCR products were gel purified using the BioSpin Gel Extraction Kit (Bioer Technology Co., Ltd) and cloned into pJET1.2 vector supplied with the CloneJET PCR cloning kit (Fermentas) and sequenced from the two ends using BigDye reagent in ABI3700 sequencer (Applied BioSystems).

\section{Phylogenetic tree construction and bioinformatics}

The $M K K s$ and $M A P K s / M P K s$ of Arabidopsis and rice (Oryza sativa subsp. japonica) were downloaded from TAIR10 (www.arabidopsis.org) and the rice genome annotation project http://rice.plantbiology.msu.edu/ annotation_community_families.shtml [1], respectively. To identify $M K K s$ and $M A P K s$ from other species, we first aligned the amino acid sequences of 10 AtMKKs and 20 AtMPKs and generated a hidden Markov model (HMM) for each. Next we performed an HMM-based search (http://hmmer.janelia.org/) [83], for similar peptide sequences in the sequenced genomes as presented in Phytozome v9.0 (http://www.phytozome.net/) and also by keyword search in the NCBI database. After that, each retrieved sequence was examined for the conserved MKK and MAPK signature motif sequences. The species searched include Brachypodium distachyon (Bd); Capsicum annuum (Ca); Chlamydomonas reinhartdii $(C r)$; Euphorbia esula (Ee); Gossypium hirsutum (Gh); Glycine max (Gm); Hordeum vulgare (Hv); Ipomoea batatas (Lb); Malus micromalus $(\mathrm{Mm})$; Medicago sativa $(\mathrm{Ms})$; Nicotiana tabacum $(\mathrm{Nb})$; Ostreococcus tauri $(\mathrm{Ot})$; Petroselinum crispum (Pc); Physcomitrella patens (Pp); Pisum sativum (Ps); Populus trichocarpa (Pt); Ricinus communis (Rc); Selaginella moellendorffii (Sm); Sorghum bicolor (Sb); Solanum lycopersicum (Sl); Saccharum officinarum (So); Solanum tuberosum (St); Triticum aestivum (Ta); Vitis vinifera $(\mathrm{Vv})$ and Zea mays $(\mathrm{Zm})$.

The phylogenetic trees of both MKKs and MPKs across various species were produced as described previously [44]. First, the predicted amino acid sequences of MKKs or MPKs were aligned using ClustalX1.83. Then, by using the maximum parsimony (MP) algorithm implemented in MEGA5.1 [84], the bootstrap consensus trees were inferred from 500 replicates to represent the inferred evolutionary history. The amino acid sequences of MKKs or MPKs from a variety of species retrieved from online databases were also identified for the reconstruction of the phylogenetic trees.

We analyzed the predicted candidate proteins of BnaMKK and BnaMPK gene families using MEME 4.9.0 (Release date: Wed Oct 3 11:07:26 EST 2012) with the parameters as described previously $[47,85]$. The maximum number of motifs was set to 10 and the optimum motif width was 6 to 50. For prediction of transactivation domain (TAD) of transcription factors, a 9aa TAD prediction program was used (http://www.at.embnet. org/toolbox/9aatad).

\section{Subcellular localization and confocal microscopy}

The coding regions (CDSs) of BnaMKK2, -3, -4 and BnaMPK-3, -5, -6, and -9, were amplified by RT-PCR from the CDNA of canola with primers listed (Additional File 9: Table S4). Both pCsGFPBT (GenBank: DQ370426) binary vector and a modified version, pYJGFP with a Gly-Ala rich peptide linker between CDS and sGFP were used in this study [67]. The modified GFP vector, pYJGFP was made by insertion of multiple cloning sites at Nco I site and sequenced to confirm that it was in-frame. These vectors and $B n a M K K$ or BnaMPK products were digested with corresponding restriction enzymes (Ferments), purified (Bioer) and ligated by $\mathrm{T}_{4}$ DNA ligase (Fermentas) before transformed into DH5 $\alpha$. After confirmed by sequencing, these constructs and p19 protein of tomato bushy stunt virus were transferred into Agrobacterium tumefaciens GV3101 for infiltrating into the leaves of $N$. benthamiana [86]. P19 can suppress gene silencing in transformed tobacco leaves. AS-media (for $100 \mathrm{ml}, 1 \mathrm{ml}$ of $1 \mathrm{M}$ MES$\mathrm{KOH}, \mathrm{pH} 5.6,333 \mu \mathrm{l}$ of $3 \mathrm{M} \mathrm{MgCl}_{2}, 100 \mu \mathrm{l}$ of $150 \mathrm{mM}$ acetosyringon [in DMSO, stored in aliquots at $-20^{\circ} \mathrm{C}$ ]) was freshly made to resuspend transformed agrobacteria. One mililiter of the culture was taken by sterile single-use syringes to inject into the abaxial air space of tobacco leaves. The leaf section near the injection site was taken and $500 \mathrm{mM}$ mannitol was used to treat leaves section for one hour at room temperature to separate the cell wall and protoplast. Observation of GFP signal was performed under a confocal microscope LSM510beta (Carl Zeiss). 


\section{Fungal pathogen inoculation and stress treatments}

Wild type canola plants were grown in a greenhouse with a photoperiod of $16 \mathrm{~h}$ light $/ 8 \mathrm{~h}$ dark for $18 \mathrm{~d}$. $S$. sclerotiorum (SX09-904) was a gift provided by Prof. Lili Huang (Northwest A \& F University) and was cultured on Potato Dextrose Agar (PDA) mdium. Agar plugs from the front edge of mycelia were used to inoculate the first and the second true leaves with wounding. Leaves inoculated with agar plugs without mycelia were used as mock control. Tissues were harvested at 6 and $24 \mathrm{~h}$ post inoculation (hpi) and flash frozen in liquid nitrogen before stored at $-80^{\circ} \mathrm{C}$. Jasmonic acid (JA, SigmaAldrich, USA), SA (Sigma-Aldrich, USA), Paraquat (MV, Sigma-Aldrich), abscisic acid (( \pm$)$-ABA, Invitrogen, USA), 1-aminocyclopropane-1-carboxylic acid (ACC, Sigma-Aldrich), oxalic acid (OA, Sigma-Aldrich) were sprayed onto the leaves with a concentration of $100 \mu \mathrm{M}$ for JA, $2 \mathrm{mM}$ for SA, $50 \mu \mathrm{M}$ for ABA, $25 \mu \mathrm{M}$ for ACC, $10 \mu \mathrm{M}$ for Paraquat and $40 \mathrm{mM}$ for OA. The preparation of stock solution of JA, SA, and ACC followed our previous procedures [44], while OA was prepared as a $40 \mathrm{mM}$ stock solution as described in [87]. The whole process was repeated three times at independently time and thus three biological replicates were prepared.

\section{Quantitative RT-PCR (qRT-PCR)}

The quantitative reverse transcriptase PCR (qRT-PCR) was performed as described earlier [44] with modifications. Total RNA samples were isolated from treated and non-treated canola leaves using Plant RNA kit (Omega, USA), quantified by NanoDrop (NanoDrop Technologies, Inc.) with the integrity checked on $1 \%(\mathrm{w} / \mathrm{v})$ agarose gel. RNA samples were transcribed into cDNAs by using RevertAid $\mathrm{H}$ minus reverse transcriptase (Fermentas,) and oligo $(\mathrm{dT})_{18}$ primers (Fermentas). Primers used for qRT-PCR were designed using PrimerSelect program of DNASTAR (DNASTAR Inc.) targeting 3'UTR of each genes with amplicon sizes of 75-250 bp (Additional file 9: Table S4). Each primer was reciprocally BLAST against the B. napus database in NCBI to eliminate crossamplification. Primer specificity and amplification efficiency were further checked by running standard curves with melting curves examined. Three independent biological replicates were run and the significance was determined with SPSS software $(\mathrm{p}<0.05)$.

\section{Yeast two-hybrid assay}

Yeast two-hybrid ( $\mathrm{Y} 2 \mathrm{H})$ analysis was performed in accordance with the Yeast Protocols Handbook (Clontech, USA). The coding regions of BnaMKK, BnaMPK and BnaWRKY genes were amplified from cDNA fragments of canola by high-fidelity PCR, restricted and cloned into pGBKT7 (BnaMPKs) or pGADT7 (BnaMKKs, $B n a W R K Y$ s) vectors, respectively, before transformed into yeast strain AH109 through the lithium acetate method. After selection on media (SD-Leucine-Tryptophan, SD-LT; SD-Leucine-Tryptophan-Histidine + 3'AT, [SD-LTH + 3'AT]; SD-Adenine-Histidine-Leucine-Tryptophan, SD-LTHA), the positive interaction transformants were cultivated into Yeast, peptone, dextrose (YEPD) media for serial dilution. The exponentially grown yeast cells were centrifuged at $3000 \mathrm{~g}$ at room temperature and adjusted to $\mathrm{OD}_{600}=0.5$ with sterilized double-distilled water. Later on, it was diluted 1/10, 1 / 100 and 1/1000. Two microliters of the aforementioned serial diluted yeast cells were spotted onto SD-LT, SD-LTH + 3'AT and SD-LTHA media, grown in 30C for 2-5 days, before photographed.

The colony-lift filter assay was conducted following the instruction in the Yeast Protocols Handbook. The freshly grown colonies on the selection media SD-LTHA were transferred onto sterilized $9 \mathrm{~cm}$ filter papers and then flash frozen in liquid nitrogen for 20 seconds. After thawed completely, the filter paper with colonies side up was transferred onto presoaked filter paper in $5 \mathrm{ml}$ of staining buffer $\left(60 \mathrm{mM} \mathrm{Na}_{2} \mathrm{HPO}_{4}, 39.8 \mathrm{mM} \mathrm{NaH} \mathrm{PO}_{4}\right.$, $10 \mathrm{mM} \mathrm{KCl}, 1 \mathrm{mM} \mathrm{MgSO} 4,0.817 \mathrm{mM}$ 5-bromo4-chloro-3-indolyl- $\beta$-D-galactopyranoside [X-gal] and $38.5 \mathrm{mM} \beta$-mercaptomethanol) in a $90 \mathrm{~mm}$ petri dish for $8-12 \mathrm{~h}$ at $37^{\circ} \mathrm{C}$. After that, the reaction was stopped and filter paper dried before photographed.

\section{In vivo bimolecular florescence complementation (BiFC) analysis}

For yellow fluorescence protein (YFP)-based BiFC, the coding regions of tested BnaMPKs were subcloned into pSPYNE(R)173 and, the coding regions of BnaMKK or BnaWRKY genes were subcloned into $\mathrm{PSPYCE}(\mathrm{M})$ vector [88]. The primers used are listed in Additional file 9: Table S4. After confirmation by sequencing, these constructs and p19 of tomato bushy stunt virus were transferred into A. tumefaciens GV3101. Freshly grown agrobacterium cultures were resuspended in a freshly made solution containing $10 \mathrm{mM}$ MES-KOH, pH 5.6, $10 \mathrm{mM} \mathrm{MgCl}_{2}$ and $0.15 \mathrm{mM}$ acetosyringone, with $\mathrm{OD}_{600}$ adjusted to be $0.7-0.8$, before mixed at 1:1:1 ratio, incubated at room temperature for 2-4 h, and infiltrated into the leaves of $N$. benthamiana [89]. At $4 \mathrm{~d}$, the leaf sections near the injection sites were taken and used to examine the YFP signals under a confocal microscope A1R (Nikon, Japan).

\section{Additional files}

Additional file 1: Table S1. BnaMPK and BnaMKK EST summary. Additional file 2: Table S2. MKK and MPK sequences from different species used for phylogenetic analysis. 
Additional file 3: Figure S1. Phylogenetic analysis of MKKs from a variety of species.

Additional file 4: Figure S2. A detailed motif analysis and multiple alignment of canola MKKs.

Additional file 5: Figure S3. Phylogenetic analysis of MPKs from a variety of species.

Additional file 6: Figure S4. Multiple alignment of MAPK domains of BnaMPKs and selected Arabidopsis, rice, B. distachyon and O. tauri MAPKs.

Additional file 7: Figure S5. A detailed motif analysis and multiple alignment of BnaMPKs.

Additional file 8: Table S3. Arabidopsis MPK and MKK expression profiles in response to different stresses. GENEVESTIGATOR database was used to analyze the gene expression levels.

Additional file 9: Table S4. Primers used in this study.

\section{Competing interests}

The authors are not aware of any potent competing interests.

\section{Authors' contributions}

Conceived and designed the experiment: $Y Q \mathrm{Q} J$ and BY. Performed the experiments: BY, YQJ, WWL, BJY, ZZ, CL, YS, YZ, MJ, FFW, HZ and BW. Analyzed the data: BY, YQJ, WWL and MKD. Provided material: MKD. Wrote the manuscript: BY, MKD and YQJ. All authors read and approved the manuscript.

\section{Acknowledgements}

We would like to thank Profs. X-H Shen, K-M Chen, T-Y Zhao and Y Wang for facility use. We also thank Prof. Lili Huang for providing Sclerotinia scleortiorum strain of SX09-904 and Prof. Jörg Kudla (Universität Münster, Germany) for providing the BiFC vectors. This work was supported by the National Natural Science Foundation of China (No. 31270293 to YOJ) and Chinese Ministry of Education Program for New Teachers in Universities (No. 20110204120005 to BY).

\section{Author details}

'State Key Laboratory of Crop Stress Biology for Arid Areas and College of Life Sciences, Northwest A \& F University, Yangling, Shaanxi 712100, China. ${ }^{2}$ Department of Biological Sciences, University of Alberta, Edmonton T6G 2E9, Canada.

Received: 22 February 2013 Accepted: 21 May 2013

Published: 11 June 2013

\section{References}

1. Hamel LP, Nicole MC, Sritubtim S, Morency MJ, Ellis M, Ehlting J, Beaudoin N, Barbazuk B, Klessig D, Lee J, et al: Ancient signals: comparative genomics of plant MAPK and MAPKK gene families. Trends Plant Sci 2006, 11:192-198.

2. Rodriguez $M C$, Petersen $M$, Mundy J: Mitogen-activated protein kinase signaling in plants. Annu Rev Plant Biol 2010, 61:621-649.

3. Jonak C, Okresz L, Bogre L, Hirt H: Complexity, cross talk and integration of plant MAP kinase signalling. Curr Opin Plant Biol 2002, 5:415-424.

4. Ichimura K, Shinozaki K, Tena G, Sheen J, Henry Y, Champion A, Kreis M, Zhang S, Hirt H, Wilson C, et al: Mitogen-activated protein kinase cascades in plants: a new nomenclature. Trends Plant Sci 2002, 7:301-308.

5. Gao M, Liu J, Bi D, Zhang Z, Cheng F, Chen S, Zhang Y: MEKK1, MKK1/ MKK2 and MPK4 function together in a mitogen-activated protein kinase cascade to regulate innate immunity in plants. Cell Res 2008 18:1190-1198.

6. Teige M, Scheikl E, Eulgem T, Doczi R, Ichimura K, Shinozaki K, Dangl JL, Hirt H: The MKK2 pathway mediates cold and salt stress signaling in Arabidopsis. Mol Cell 2004, 15:141-152.

7. Asai T, Tena G, Plotnikova J, Willmann MR, Chiu WL, Gomez-Gomez L, Boller T, Ausubel FM, Sheen J: MAP kinase signalling cascade in Arabidopsis innate immunity. Nature 2002, 415:977-983.

8. Matsuoka D, Nanmori T, Sato K, Fukami Y, Kikkawa U, Yasuda T: Activation of AtMEK1, an Arabidopsis mitogen-activated protein kinase kinase, in vitro and in vivo: analysis of active mutants expressed in E. coli and generation of the active form in stress response in seedlings. Plant J 2002, 29:637-647.

9. Ueda M, Zhang Z, Laux T: Transcriptional activation of Arabidopsis axis patterning genes WOX8/9 links zygote polarity to embryo development. Dev Cell 2011, 20:264-270.

10. Jeong S, Palmer TM, Lukowitz W: The RWP-RK factor GROUNDED promotes embryonic polarity by facilitating YODA MAP kinase signaling. Curr Biol 2011, 21:1268-1276.

11. Wang Z, Mao H, Dong C, Ji R, Cai L, Fu H, Liu S: Overexpression of Brassica napus MPK4 enhances resistance to Sclerotinia sclerotiorum in oilseed rape. Mol Plant Microbe Interact 2009, 22:235-244.

12. Qiu JL, Fiil BK, Petersen K, Nielsen HB, Botanga CJ, Thorgrimsen S, Palma K, Suarez-Rodriguez MC, Sandbech-Clausen S, Lichota J, et al: Arabidopsis MAP kinase 4 regulates gene expression through transcription factor release in the nucleus. Embo Journal 2008, 27:2214-2221.

13. Rushton PJ, Somssich IE, Ringler P, Shen QJ: WRKY transcription factors. Trends Plant Sci 2010, 15:247-258.

14. Ishihama N, Yoshioka H: Post-translational regulation of WRKY transcription factors in plant immunity. Curr Opin Plant Bio/ 2012, 15:431-437.

15. Suarez-Rodriguez MC, Adams-Phillips L, Liu Y, Wang H, Su SH, Jester PJ, Zhang S, Bent AF, Krysan PJ: MEKK1 is required for flg22-induced MPK4 activation in Arabidopsis plants. Plant Physiol 2007, 143:661-669.

16. Nakagami H, Soukupova H, Schikora A, Zarsky V, Hirt H: A Mitogenactivated protein kinase kinase kinase mediates reactive oxygen species homeostasis in Arabidopsis. J Biol Chem 2006, 281:38697-38704.

17. Ichimura K, Casais C, Peck S, Shinozaki K, Shirasu K: MEKK1 is required for MPK4 activation and regulates tissue-specific and temperaturedependent cell death in Arabidopsis. J Biol Chem 2006, 281:36969-36976.

18. Andreasson E, Jenkins T, Brodersen P, Thorgrimsen S, Petersen NH, Zhu S, Qiu $J$, Micheelsen P, Rocher A, Petersen M, et al: The MAP kinase substrate MKS1 is a regulator of plant defense responses. Embo J 2005, 24:2579-2589.

19. Ren D, Liu Y, Yang KY, Han L, Mao G, Glazebrook J, Zhang S: A fungal-responsive MAPK cascade regulates phytoalexin biosynthesis in Arabidopsis. Proc Natl Acad Sci U S A 2008, 105:5638-5643.

20. Mao G, Meng X, Liu Y, Zheng Z, Chen Z, Zhang S: Phosphorylation of a WRKY transcription factor by two pathogen-responsive MAPKs drives phytoalexin biosynthesis in Arabidopsis. Plant Cell 2011, 23:1639-1653.

21. Berriri S, Garcia AV, Frei Dit Frey N, Rozhon W, Pateyron S, Leonhardt N, Montillet JL, Leung J, Hirt H, Colcombet J: Constitutively active mitogenactivated protein kinase versions reveal functions of Arabidopsis MPK4 in pathogen defense signaling. Plant Cell 2012, 24:4281-4293.

22. Xing Y, Jia W, Zhang J: AtMKK1 and AtMPK6 are involved in abscisic acid and sugar signaling in Arabidopsis seed germination. Plant Mol Biol 2009. 70:725-736.

23. Li G, Meng X, Wang R, Mao G, Han L, Liu Y, Zhang S: Dual-level regulation of ACC synthase activity by MPK3/MPK6 cascade and its downstream WRKY transcription factor during ethylene induction in Arabidopsis. PLOS Genet 2012, 8:e1002767.

24. Wang H, Ngwenyama N, Liu Y, Walker JC, Zhang S: Stomatal development and patterning are regulated by environmentally responsive mitogen-activated protein kinases in Arabidopsis. Plant Cell 2007, 19:63-73.

25. Gudesblat GE, lusem ND, Morris PC: Guard cell-specific inhibition of Arabidopsis MPK3 expression causes abnormal stomatal responses to abscisic acid and hydrogen peroxide. New Phytol 2007, 173:713-721.

26. Doczi R, Brader G, Pettko-Szandtner A, Rajh I, Djamei A, Pitzschke A, Teige M, Hirt H: The Arabidopsis mitogen-activated protein kinase kinase MKK3 is upstream of group C mitogen-activated protein kinases and participates in pathogen signaling. Plant Cell 2007, 19:3266-3279.

27. Cheong YH, Moon BC, Kim JK, Kim CY, Kim MC, Kim IH, Park CY, Kim JC, Park BO, Koo SC, et al: BWMK1, a rice mitogen-activated protein kinase, locates in the nucleus and mediates pathogenesis-related gene expression by activation of a transcription factor. Plant Physiol 2003, 132:1961-1972.

28. Xiong $L$, Yang $Y$ : Disease resistance and abiotic stress tolerance in rice are inversely modulated by an abscisic acid-inducible mitogen-activated protein kinase. Plant Cell 2003, 15:745-759.

29. Xie G, Kato H, Imai R: Biochemical identification of the OsMKK6-OsMPK3 signalling pathway for chilling stress tolerance in rice. Biochem J 2012, 443:95-102. 
30. Sharma PC, Ito A, Shimizu T, Terauchi R, Kamoun S, Saitoh H: Virus-induced silencing of WIPK and SIPK genes reduces resistance to a bacterial pathogen, but has no effect on the INF1-induced hypersensitive response (HR) in Nicotiana benthamiana. Mol Genet Genomics 2003, 269:583-591.

31. Zhang S, Liu Y, Klessig DF: Multiple levels of tobacco WIPK activation during the induction of cell death by fungal elicitins. Plant J 2000 23:339-347.

32. Wang J, Ding $H$, Zhang A, Ma F, Cao J, Jiang M: A novel mitogen-activated protein kinase gene in maize (Zea mays), ZmMPK3, is involved in response to diverse environmental cues. J Integr Plant Biol 2010, 52:442-452.

33. Lin F, Ding $H$, Wang J, Zhang H, Zhang A, Zhang $Y$, Tan $M$, Dong W Jiang M: Positive feedback regulation of maize NADPH oxidase by mitogen-activated protein kinase cascade in abscisic acid signalling. J Exp Bot 2009, 60:3221-3238

34. Rudd JJ, Keon J, Hammond-Kosack KE: The wheat mitogen-activated protein kinases TaMPK3 and TaMPK6 are differentially regulated at multiple levels during compatible disease interactions with Mycosphaerella graminicola. Plant Physiol 2008, 147:802-815

35. Holley SR, Yalamanchili RD, Moura DS, Ryan CA, Stratmann JW: Convergence of signaling pathways induced by systemin, oligosaccharide elicitors, and ultraviolet-B radiation at the level of mitogen-activated protein kinases in Lycopersicon peruvianum suspension-cultured cells. Plant Physiol 2003, 132:1728-1738.

36. Pitzschke A, Schikora A, Hirt H: MAPK cascade signalling networks in plant defence. Curr Opin Plant Biol 2009, 12:421-426.

37. Boland GJ, Hall R: Index of plant hosts of Sclerotinia-sclerotiorum. Can J Plant Pathol 1994, 16:93-108.

38. Wang Z, Tan X, Zhang Z, Gu S, Li GJ, Shi H: Defense to Sclerotinia sclerotiorum in oilseed rape is associated with the sequential activations of salicylic acid signaling and jasmonic acid signaling. Plant Science 2012, 184:75-82.

39. Rietz S, Bernsdorff FE, Cai D: Members of the germin-like protein family in Brassica napus are candidates for the initiation of an oxidative burst that impedes pathogenesis of Sclerotinia sclerotiorum. J Exp Bot 2012, 63:5507-5519.

40. Partridge-Telenko DE, Hu J, Livingstone DM, Shew B, Phipps P, Grabau E: Sclerotinia blight resistance in Virginia-type peanut transformed with a barley oxalate oxidase gene. Phytopathology 2011, 101:786-793.

41. Yu X, Li B, Fu YP, Jiang DH, Ghabrial SA, Li GQ, Peng YL, Xie JT, Cheng JS, Huang JB, Yi XH: A geminivirus-related DNA mycovirus that confers hypovirulence to a plant pathogenic fungus. Proc Natl Acad Sci U S A 2010, 107:8387-8392.

42. Ren L, Li G, Jiang D: Characterization of some culture factors affecting oxalate degradation by the mycoparasite Coniothyrium minitans. J App Microbiol 2010, 108:173-180.

43. Yang B, Srivastava S, Deyholos MK, Kav NNV: Transcriptional profiling of canola (Brassica napus L.) responses to the fungal pathogen Sclerotinia sclerotiorum. Plant Science 2007, 173:156-171.

44. Yang B, Jiang Y, Rahman MH, Deyholos MK, Kav NN: Identification and expression analysis of WRKY transcription factor genes in canola (Brassica napus L.) in response to fungal pathogens and hormone treatments. BMC Plant Biol 2009, 9:68

45. Koonin EV: Orthologs, paralogs, and evolutionary genomics. Annu Rev Genet 2005, 39:309-338

46. Derelle E, Ferraz C, Rombauts S, Rouze P, Worden AZ, Robbens S, Partensky F, Degroeve S, Echeynie S, Cooke R, et al: Genome analysis of the smallest free-living eukaryote Ostreococcus tauri unveils many unique features. Proc Natl Acad Sci U S A 2006, 103:11647-11652.

47. Chen L, Hu W, Tan S, Wang M, Ma Z, Zhou S, Deng X, Zhang Y, Huang C, Yang G, He G: Genome-wide identification and analysis of MAPK and MAPKK gene families in Brachypodium distachyon. PLoS One 2012, 7:e46744.

48. Liu Q, Xue Q: Computational identification and phylogenetic analysis of the MAPK gene family in Oryza sativa. Plant Physiol Biochem 2007, 45:6-14.

49. Banks JA, Nishiyama T, Hasebe M, Bowman JL, Gribskov M, dePamphilis C, Albert VA, Aono N, Aoyama T, Ambrose BA, et al: The Selaginella genome identifies genetic changes associated with the evolution of vascular plants. Science 2011, 332:960-963.

50. Doczi R, Okresz L, Romero AE, Paccanaro A, Bogre L: Exploring the evolutionary path of plant MAPK networks. Trends Plant Sci 2012, 17:518-525.
51. Janitza P, Ullrich KK, Quint M: Toward a comprehensive phylogenetic reconstruction of the evolutionary history of mitogen-activated protein kinases in the plant kingdom. Front Plant Sci 2012, 3:271

52. Tanoue T, Adachi M, Moriguchi T, Nishida E: A conserved docking motif in MAP kinases common to substrates, activators and regulators. Nat Cell Biol 2000, 2:110-116.

53. Harding A, Tian T, Westbury E, Frische E, Hancock JF: Subcellular localization determines MAP kinase signal output. Curr Biol 2005, 15:869-873.

54. Samajova O, Komis G, Samaj J: Emerging topics in the cell biology of mitogen-activated protein kinases. Trends Plant Sci 2013, 18:140-148

55. Ishihama N, Yamada R, Yoshioka M, Katou S, Yoshioka H: Phosphorylation of the Nicotiana benthamiana WRKY8 transcription factor by MAPK functions in the defense response. Plant Cell 2011, 23:1153-1170.

56. Lee J, Huh K, Bhargava A, Ellis B: Comprehensive analysis of protein-protein interactions between Arabidopsis MAPKs and MAPK kinases helps define potential MAPK signalling modules. Plant Signal Behav 2008, 3:1037-1041.

57. Popescu S, Popescu G, Bachan S, Zhang Z, Gerstein M, Snyder M, Dinesh-Kumar S: MAPK target networks in Arabidopsis thaliana revealed using functional protein microarrays. Genes \& Development 2009, 23:80-92.

58. Miao Y, Laun TM, Smykowski A, Zentgraf U: Arabidopsis MEKK1 can take a short cut: it can directly interact with senescence-related WRKY53 transcription factor on the protein level and can bind to its promoter Plant Mol Biol 2007, 65:63-76.

59. Shang J, Xi DH, Xu F, Wang SD, Cao S, Xu MY, Zhao PP, Wang JH, Jia SD, Zhang ZW, et al: A broad-spectrum, efficient and nontransgenic approach to control plant viruses by application of salicylic acid and jasmonic acid. Planta 2011, 233:299-308.

60. Miao Y, Smykowski A, Zentgraf U: A novel upstream regulator of WRKY53 transcription during leaf senescence in Arabidopsis thaliana. Plant Biol (Stuttg) 2008, 10(Suppl 1):110-120.

61. Murray SL, Ingle RA, Petersen LN, Denby KJ: Basal resistance against Pseudomonas syringae in Arabidopsis involves WRKY53 and a protein with homology to a nematode resistance protein. Mol Plant Microbe Interact 2007, 20:1431-1438.

62. Xu J, Li Y, Wang Y, Liu H, Lei L, Yang H, Liu G, Ren D: Activation of MAPK kinase 9 induces ethylene and camalexin biosynthesis and enhances sensitivity to salt stress in Arabidopsis. J Biol Chem 2008, 283:26996-27006.

63. Zhou C, Cai Z, Guo Y, Gan S: An arabidopsis mitogen-activated protein kinase cascade, MKK9-MPK6, plays a role in leaf senescence. Plant Physiol 2009, 150:167-177.

64. Yoo SD, Cho YH, Tena G, Xiong Y, Sheen J: Dual control of nuclear EIN3 by bifurcate MAPK cascades in C2H4 signalling. Nature 2008, 451:789-795.

65. Brader G, Djamei A, Teige M, Palva ET, Hirt H: The MAP kinase kinase MKK2 affects disease resistance in Arabidopsis. Mol Plant Microbe Interact 2007, 20:589-596.

66. Remenyi A, Good MC, Lim WA: Docking interactions in protein kinase and phosphatase networks. Curr Opin Struct Biol 2006, 16:676-685.

67. Jiang Y, Deyholos MK: Functional characterization of Arabidopsis $\mathrm{NaCl}$-inducible WRKY25 and WRKY33 transcription factors in abiotic stresses. Plant Mol Biol 2009, 69:91-105.

68. Li S, Fu Q, Chen L, Huang W, Yu D: Arabidopsis thaliana WRKY25, WRKY26, and WRKY33 coordinate induction of plant thermotolerance. Planta 2011, 233:1237-1252.

69. Gao X, Chen X, Lin W, Chen S, Lu D, Niu Y, Li L, Cheng C, McCormack M, Sheen J, et al: Bifurcation of Arabidopsis NLR Immune Signaling via $\mathrm{Ca}(2+)$-Dependent Protein Kinases. PLoS Pathog 2013, 9:e1003127.

70. Singh R, Lee MO, Lee JE, Choi J, Park JH, Kim EH, Yoo RH, Cho Jl, Jeon JS, Rakwal R, Agrawal GK, Moon JS, Jwa NS: Rice mitogen-activated protein kinase interactome analysis using the yeast two-hybrid system. Plant Physiol 2012, 160:477-487.

71. Xu X, Chen C, Fan B, Chen Z: Physical and functional interactions between pathogen-induced Arabidopsis WRKY18, WRKY40, and WRKY60 transcription factors. Plant Cell 2006, 18:1310-1326.

72. Samajova O, Plihal O, Al-Yousif M, Hirt H, Samaj J: Improvement of stress tolerance in plants by genetic manipulation of mitogen-activated protein kinases. Biotechnol Adv 2011, 31:118-128.

73. Farmer EE, Almeras E, Krishnamurthy V: Jasmonates and related oxylipins in plant responses to pathogenesis and herbivory. Curr Opin Plant Biol 2003, 6:372-378. 
74. Vlot AC, Dempsey DA, Klessig DF: Salicylic Acid, a multifaceted hormone to combat disease. Annu Rev Phytopathol 2009, 47:177-206.

75. Ton J, Flors V, Mauch-Mani B: The multifaceted role of ABA in disease resistance. Trends Plant Sci 2009, 14:310-317.

76. van Loon LC, Geraats BP, Linthorst HJ: Ethylene as a modulator of disease resistance in plants. Trends Plant Sci 2006, 11:184-191.

77. Guimaraes RL, Stotz HU: Oxalate production by Sclerotinia sclerotiorum deregulates guard cells during infection. Plant Physiol 2004, 136:3703-3711.

78. Williams B, Kabbage M, Kim HJ, Britt R, Dickman MB: Tipping the balance: Sclerotinia sclerotiorum secreted oxalic acid suppresses host defenses by manipulating the host redox environment. Plos Pathogens 2011, 7:e1002107.

79. Cessna SG, Sears VE, Dickman MB, Low PS: Oxalic acid, a pathogenicity factor for Sclerotinia sclerotiorum, suppresses the oxidative burst of the host plant. Plant Cell 2000, 12:2191-2200.

80. Jin ZX, Wang C, Chen W, Chen X, Li X: Induction of oxalate decarboxylase by oxalate in a newly isolated Pandoraea sp. OXJ-11 and its ability to protect against Sclerotinia sclerotiorum infection. Can J Microbiol 2007, 53:1316-1322

81. Rietz S, Bernsdorff FE, Cai D: Members of the germin-like protein family in Brassica napus are candidates for the initiation of an oxidative burst that impedes pathogenesis of Sclerotinia sclerotiorum. J Exp Bot 2008, 63:5507-5519.

82. Takahashi F, Yoshida R, lchimura K, Mizoguchi T, Seo S, Yonezawa M, Maruyama K, Yamaguchi-Shinozaki K, Shinozaki K: The mitogen-activated protein kinase cascade MKK3-MPK6 is an important part of the jasmonate signal transduction pathway in Arabidopsis. Plant Cell 2007, 19:805-818.

83. Finn RD, Clements J, Eddy SR: HMMER web server: interactive sequence similarity searching. Nucleic Acids Res 2011, 39:W29-W37.

84. Tamura K, Peterson D, Peterson N, Stecher G, Nei M, Kumar S: MEGA5: Molecular Evolutionary Genetics Analysis using Maximum Likelihood, Evolutionary Distance, and Maximum Parsimony Methods. Mol Biol Evol 2011, 28:2731-2739.

85. Bailey $T L$, Elkan C: Fitting a mixture model by expectation maximization to discover motifs in biopolymers. Proc Int Conf Intell Syst Mol Biol 1994:28-36.

86. Sparkes IA, Runions J, Kearns A, Hawes C: Rapid, transient expression of fluorescent fusion proteins in tobacco plants and generation of stably transformed plants. Nat Protoc 2006, 1:2019-2025.

87. Liang Y, Srivastava S, Rahman M, Strelkov S, Kav NN: Proteome changes in leaves of Brassica napus L. as a result of Sclerotinia sclerotiorum challenge. J Agric Food Chem 2008, 56:1963-1976.

88. Waadt R, Schmidt LK, Lohse M, Hashimoto K, Bock R, Kudla J: Multicolor bimolecular fluorescence complementation reveals simultaneous formation of alternative CBL/CIPK complexes in planta. Plant J 2008, 56:505-516

89. Waadt R, Kudla J: In Planta Visualization of Protein Interactions Using Bimolecular Fluorescence Complementation (BiFC). CSH Protoc 2008:pdb prot4995.

doi:10.1186/1471-2164-14-392

Cite this article as: Liang et al: Identification and analysis of MKK and MPK gene families in canola (Brassica napus L.). BMC Genomics 2013 14:392.

\section{Submit your next manuscript to BioMed Central and take full advantage of:}

- Convenient online submission

- Thorough peer review

- No space constraints or color figure charges

- Immediate publication on acceptance

- Inclusion in PubMed, CAS, Scopus and Google Scholar

- Research which is freely available for redistribution 